\title{
Revised and extended analysis of the eighth spectrum of platinum (Pt VIII)
}

\author{
Vladimir I. Azarov ${ }^{1}$ and Robert R. Gayasov ${ }^{2}$ \\ ${ }^{1}$ National Institute of Standards and Technology, Gaithersburg, MD 20899, USA. \\ E-mail:vlad_azarov@yahoo.com \\ ${ }^{2}$ Institute of Spectroscopy, Russian Academy of Sciences, Troitsk, Moscow, 108840 Russian \\ Federation.
}

\begin{abstract}
The spectrum of platinum was observed in the 300-2100 $\AA$ wavelength region. Grazing and normal incidence VUV spectrographs have been used to record the spectrum. The $\left(5 d^{3}+5 d^{2} 6 s\right)-$ $5 \mathrm{~d}^{2} 6 \mathrm{p}$ transition array of seven times ionized platinum, Pt VIII, has been investigated. The configurations $5 \mathrm{~d}^{3}$ and $5 \mathrm{~d}^{2} 6 \mathrm{p}$ had been previously studied, and all levels of these configurations (19 and 45 levels, respectively) had been established. The previous analysis was based on 178 classified spectral lines. In the current analysis we have confirmed identification of all previously found levels and all but 10 previously assigned spectral lines, although we have detected a large (up to $35 \mathrm{~m} \AA$ ) systematic shift in wavelength measurements used in the previous analysis. Based on new wavelength measurements, we have corrected the $5 \mathrm{~d}^{3}$ and $5 \mathrm{~d}^{2} 6 \mathrm{p}$ energy level values (by up to $55 \mathrm{~cm}^{-1}$ ) and established for the first time 14 out of 16 theoretically possible $5 \mathrm{~d}^{2} 6 \mathrm{~s}$ levels in Pt VIII. The total list of identified lines (including 180 new lines) contains 349 entries. The orthogonal operators technique was used to calculate the level structure and transition probabilities. The energy parameters have been determined by the least squares fit to the observed levels. Calculated transition probability and energy values, as well as $L S$-compositions obtained from the fitted parameters are presented.
\end{abstract}

PACS Ref: 32.30.-r, 32.30.Jc

Keywords: spectral line identification, energy level, atomic spectrum, vacuum ultraviolet, seven times ionized platinum, $\mathrm{Pt}^{7+}$.

\section{Contents}

1. Introduction and experiment 2

2. Level optimization and reliability check $\quad 2$

3. Analysis 3

4. Calculations $\quad 5$

Acknowledgements $\quad 6$

References $\quad 6$

Explanation of Tables $\quad 6$

Tables 9

Table 1. Classified lines in the $\left(5 d^{3}+5 d^{2} 6 s\right)-5 d^{2} 6 p$ transition array of Pt VIII

Table 2.Experimental and calculated energy levels $\left(\mathrm{cm}^{-1}\right)$ in the $5 \mathrm{~d}^{3}$ and $5 \mathrm{~d}^{2} 6 \mathrm{~s}$ configurations of Pt VIII

Table 3. Experimental and calculated energy levels $\left(\mathrm{cm}^{-1}\right)$ in the $5 \mathrm{~d}^{2} 6 \mathrm{p}$

configuration of Pt VIII

Table 4. Fitted and calculated parameter values $\left(\mathrm{cm}^{-1}\right)$ in the $5 \mathrm{~d}^{3}, 5 \mathrm{~d}^{2} 6 \mathrm{~s}$ and $5 \mathrm{~d} 6 \mathrm{~s}^{2}$ 
configurations of Pt VIII

Table 5. Fitted and calculated parameter values $\left(\mathrm{cm}^{-1}\right)$ in the $5 \mathrm{~d}^{2} 6 \mathrm{p}, 5 \mathrm{~d} 6 \mathrm{~s} 6 \mathrm{p}$ and

$6 s^{2} 6 p$ configurations of Pt VIII

\section{Introduction and experiment}

The first analysis of the Pt VIII spectrum was published in 2002 [1]. It has resulted in establishing all levels of the configurations $5 \mathrm{~d}^{3}$ and $5 \mathrm{~d}^{2} 6 \mathrm{p}$. One hundred seventy eight lines have been identified as transitions between levels of these configurations. These two configurations are known in the following spectra of the Lu I isoelectronic sequence: Ta III, W IV, Re V, Os VI, Ir VII, Pt VIII, Au IX and Hg X [1-8]. In the sequence, the configuration $5 \mathrm{~d}^{2} 6 \mathrm{~s}$ has been studied in the spectra from Ta III till Ir VII [2-5,7]. The purpose of the present work is to determine levels of the configuration $5 \mathrm{~d}^{2} 6 \mathrm{~s}$ in Pt VIII, which were never studied before.

The spectrograms for the current analysis have been photographed in the wavelength region of 300$2100 \AA$ at the Institute of Spectroscopy, Troitsk, Moscow, Russian Federation. The spectra in the wavelength region below $340 \AA$ were recorded on a $3 \mathrm{~m}$ grazing incidence spectrograph with a plate factor of about $0.4 \AA / \mathrm{mm}$ to $0.5 \AA / \mathrm{mm}$. Lines of Ti V - Ti XI were used as the reference lines in the region. The spectra in the wavelength region above $340 \AA$ were obtained on a $6.65 \mathrm{~m}$ normal incidence spectrograph with a plate factor of $1.25 \AA / \mathrm{mm}$. For this region, impurity lines of $\mathrm{O}, \mathrm{C}, \mathrm{N}, \mathrm{Al}$ and $\mathrm{Si}$ [9] present in the spectrograms were used as standards in the initial wavelength interpolation procedure. Details of the experimental setup can be found in Ref. [1]. The photographic plates were measured on an automatic comparator controlled by the system for automatic processing of photo-spectrograms [10]. Lines belonging to different ionization stages could be discriminated on our plates on tracks exposed with different voltages and currents in the sliding and triggered sparks that were used as light sources. Our spectrograms contain Pt lines appearing in the second order. Presence of these lines has allowed us to improve wavelength measurements by using secondary standards derived from the second-order lines. Since wavelengths of spectral lines measured in the first and second orders should match, adding such correlated pairs of lines in the wavelength interpolation procedure provides additional constraints for the calibration curve. Good example is when one line of a pair is in the region with many reliable standards and the second line is in the region where there are no standards or they have large uncertainty. In this case, the second line (with wavelength derived from the first line) may be used as a reference line. The use of internally derived Ritz standards also contributed to improvement of the measurements. Such standards have been obtained in the course of simultaneous analyses of Pt VIII, Pt VII and Pt VI (both to be published), Pt V[11], and Pt IV[12] spectra. The final list of identified Pt VIII lines shows standard deviations of isolated lines from the calculated positions of less than $0.003 \AA$ and $0.004 \AA$ for the regions of 298-930 $\AA$ and 940-1150 $\AA$, respectively. The lines resolved in the second order have been measured more accurately.

We have found that wavelength measurements in the region of 337-400 $\AA$ used in the previous analysis [1] have significant systematic shift rising gradually from $10 \mathrm{~m} \AA$ to $35 \mathrm{~m} \AA$ as wavelength goes from $337 \AA$ to $400 \AA$. Comparing with the previous study, in the present work, for the region shorter than $337 \AA$, we used the same measurements. For the region longer than $337 \AA$, we used different spectrogram obtained with the same experimental set up.

The analysis presented here was based on theoretical calculations utilizing the orthogonal operators approach [13-16] and performed using the program suite IDEN $[17,18]$.

\section{Level optimization and reliability check}

The IDEN computer program [17,18] includes an iterative level optimization procedure (LOP) [19] for optimization of the energy level system comprising specified identified levels and spectral lines. For each established level, the code provides two types of uncertainties of the level with respect to the levels directly connected to it by the identified transitions. These uncertainties are suitable for the verification of the reliability and accuracy of the obtained level. The first type of uncertainty is entirely based on the specified estimated uncertainties of the experimental wavelengths. It is a square root of the weighted mean variance (WMV) of the energy level given by Eq. (7) of Ref. [19]. It is referred to as $\mathrm{rwmv}_{1}$. The second type of 
uncertainty is calculated by re-estimating uncertainties of the involved experimental wavelengths accounting for the deviations of these wavelengths from the calculated (Ritz) values. It is a square root of the WMV of the energy level given by Eq. (8) of Ref. [19]. It is referred to as $\mathrm{rwmv}_{2}$. We exclude from the optimization all levels determined by a single transition. If a spectral line is considered to be masked, blended or its identification is doubtful, the line is not included in the LOP. If the uncertainties of wavelengths of the spectral lines used in the LOP are estimated correctly and all disturbed lines are removed from the optimization, we expect the values given by Eq. (8) of Ref. [19] to be close to or smaller than those given by Eq. (7) of Ref. [19]. We verify this relation for each level and use it as a quality check of the LOP results and the entire analysis. All uncertainties given in this paper are at the level of one standard deviation.

\section{Analysis}

The ion $\mathrm{Pt}^{7+}$ studied here belongs to the $\mathrm{Lu}$ I isoelectronic sequence. The ground-state configuration of Pt VIII is $5 \mathrm{~d}^{3}$ and the lowest two excited configurations of different parities are $5 \mathrm{~d}^{2} 6 \mathrm{~s}$ and $5 \mathrm{~d}^{2} 6 \mathrm{p}$. The configurations $5 \mathrm{~d}^{3}$ and $5 \mathrm{~d}^{2} 6 \mathrm{p}$ are known in Pt VIII [1], as well as in Ta III, W IV, Re V, Os VI, Ir VII, Au IX and $\mathrm{Hg} X$ [2-8]. The configuration $5 \mathrm{~d}^{2} 6 \mathrm{~s}$ has been determined in the spectra from Ta III till Ir VII [2-5,7]. Parametric approximation of such amount of experimental data in one isoelectronic sequence by utilizing orthogonal operators [13-16] has made it possible to predict parameters of the configuration $5 \mathrm{~d}^{2} 6 \mathrm{~s}$ in Pt VIII very accurately. To avoid line misidentifications in the very dense platinum spectra recorded, we were simultaneously making analyses of eighth and lower ionization Pt spectra using the same sets of spectrograms containing spectra photographed under different source conditions. Such approach was helpful as arrays of spectral lines belonging to different Pt ions were overlapping, and it was difficult to distinguish lines belonging to ions of close ionization stages. Spectral line identification, determination of levels of configurations and optimization of level energies were done by using the program suite IDEN [17-19]. The final level uncertainties and uncertainties of the Ritz wavelengths presented here were calculated by the program LOPT [20].

In the region of $298-1150 \AA$, we have identified 349 spectral lines belonging to the $\left(5 \mathrm{~d}^{3}+5 \mathrm{~d}^{2} 6 \mathrm{~s}\right)$ $5 d^{2} 6 p$ transition array of Pt VIII. Seven spectral lines are doubly classified. To verify if the number of multiply classified lines is acceptable, the following estimations can be done. The most rich of lines region of 300$452 \AA$ contains about 230 identified spectral lines with an average distance between two lines of about $661 \mathrm{~m} \AA$. For our typical line widths of $25-50 \mathrm{~m} \AA$, we may expect that $4 \%$ to $8 \%$ of lines are overlapping. For 230 classified lines, it gives 9 to 18 possible blended lines. In the selected region we have six multiply identified lines, which is a quite reasonable number. Comparing with the previous Pt VIII analysis [1], the current study is based on significantly improved wavelength measurements in the region of 337-400 $\AA$. We have corrected the systematic shift (reaching $35 \mathrm{~m} \AA$ ) in the measurements that was mentioned above. Eight of the previously identified lines at 327.396, 336.766, 341.065, 365.680, 368.328, 375.244, 380.604 and $392.139 \AA$ have not been confirmed and therefore have been removed from the list. We have identified 68 new spectral lines belonging to the $5 \mathrm{~d}^{3}-5 \mathrm{~d}^{2} 6 \mathrm{p}$ transition array and changed identification of two previously classified lines at $380.5668 \AA$ and $395.1143 \AA$. All these 70 lines are marked by the symbol "N" in the table of classified lines. In the current analysis, one hundred and twelve spectral lines have been assigned to the $5 \mathrm{~d}^{2} 6 \mathrm{~s}-$ $5 \mathrm{~d}^{2} 6 \mathrm{p}$ transition array.

The list of identified spectral lines is presented in Table 1. Transition probabilities $(g A)$ have been calculated from the final fitted parameters of the investigated configurations. Intensities of the spectral lines (Int) have been obtained by measuring the plate darkening. The optical density was converted to spectral intensity and the spectral background approximated by a spline curve was subtracted. For isolated, unperturbed and unsaturated lines originating from the same upper level or from close upper levels, agreement between $g A$ and Int is good. Taking into account the Boltzmann factor, describing the decrease of population for levels with increasing energy, agreement between the presented $g A$ and Int is good throughout the entire list. In each case when the intensity of an identified spectral line is notably higher than expected, the expected value is given in Table 1. Uncertainties of the Ritz wavelengths, Unc $\lambda_{\text {calc }}$, differences between the measured and Ritz wavelengths and wavenumbers, $\Delta(\lambda)$ and $\Delta(v)$, as well as the numbers of observed transitions used to determine the odd and even level involved in the particular transition, $N_{\text {odd }}$ and $N_{\text {even }}$, are included in the table. Due to strong mixing of the $5 \mathrm{~d}^{2} 6 \mathrm{p}$ levels, their labels in the $L S$-coupling scheme are omitted in Table 1 . For 
each transition, we include a name of the linelist part ( $\mathrm{pa}-\mathrm{pd}$ ) used to specify the wavelength uncertainty (see Table A), the symbol "*" for transitions that were not used in the LOP, the symbol "N" for new $5 \mathrm{~d}^{3}-5 \mathrm{~d}^{2} 6 \mathrm{p}$ transitions added in the current analysis, expected line intensity (where appropriate, derived from the $g A$ and Int comparison), and comments for masked and blended lines, and for lines that are also identified as belonging to another ion or impurity. Transitions attributed to lines that are masked, much stronger than expected, have suspiciously large deviations $\Delta(\lambda)$, or have small $g A$ values, for which chances of wrong identification increase, were excluded from the LOP. If a known line of another ion or impurity blends the line, we specify the blending spectrum in parentheses following the "bl" symbol. If the line is also identified with a transition in another ion but does not appear as a blend in the spectrogram, we do not give the "bl" symbol, although blending is possible due to a near coincidence of wavelengths. In addition, if the line is also identified with another Pt ion (e.g., Pt VII) and, when using the same linelist, the expected intensity of that identification (e.g., 100) is lower than the observed line intensity, we give "Pt VII (100)".

It should be noted that we used two sets of plates. They may have been obtained under somewhat different experimental conditions. Parts pb, pc and pd (see Table A) correspond to three plates from the same set (same exposure). The part pa corresponds to a plate from a different set (exposure). This information is needed for correct comparison of the presented $g A$ and Int values accounting for the Boltzmann factor, $\exp (-$ $E / k T)$. Different exposures may have different $k T$ values.

In Tables 2 and 3 we present experimental and calculated energy values of the even parity levels in the $5 d^{3}$ and $5 d^{2} 6 s$ configurations and the odd parity levels in the $5 d^{2} 6 p$ configuration of Pt VIII, respectively. The uncertainties of the levels, $U n c$, are given with respect to the "base" even level at $191690.18 \mathrm{~cm}^{-1}, J=7 / 2$ (see below in the text). Uncertainty of excitation energy for any level can be estimated by combining in quadrature the given uncertainty with the uncertainty of the ground level, $1.00 \mathrm{~cm}^{-1}$.

In the present analysis, all 19 theoretically possible $5 \mathrm{~d}^{3}$ levels, and all 45 possible $5 \mathrm{~d}^{2} 6 \mathrm{p}$ levels that were established in the previous analysis have been confirmed, but their values have been adjusted. All $5 \mathrm{~d}^{3}$ previously established levels (except for the ground level) have been shifted down. The shift varies from about $-12 \mathrm{~cm}^{-1}$ to about $-55 \mathrm{~cm}^{-1}$ (see level at $75993.15 \mathrm{~cm}^{-1}, J=3 / 2$ ). The correction values for $5 \mathrm{~d}^{2} 6 \mathrm{p}$ levels are ranging from about $-30 \mathrm{~cm}^{-1}$ to about $+25 \mathrm{~cm}^{-1}$. In the current study, we have established 14 of 16 possible $5 \mathrm{~d}^{2} 6 \mathrm{~s}$ levels. Two remaining levels either produce too weak transitions to be observed on our plates or are expected to give rise to only one or two relatively weak lines that cannot be chosen without ambiguity among several possibilities.

We have divided the list of experimental lines into four parts, pa - pd (see Table 1, last column), and assigned to them initial estimated uncertainties. The assigned uncertainties are based on the available standards, distribution of the standards around the wavelength interpolation curve, and the expected increased uncertainty near ends of the plates. After correction of local systematic deviations (LSD, see Ref. [2] and [19]) in the linelist and after the final LOP run, for each part, we have calculated the root-mean-square (rms) deviation of wavelengths of the undisturbed experimental lines from their calculated (Ritz) positions. They are presented in Table A below, where for each linelist part the number of lines used in the LOP is also given.

\section{Table A}

Description of linelist parts.

\begin{tabular}{lllll}
\hline Linelist part & $\mathrm{pa}$ & $\mathrm{pb}$ & $\mathrm{pc}$ & $\mathrm{pd}$ \\
Final estimated uncertainty, $\mathrm{m} \AA$ & 3 & 3 & 3 & 4 \\
Resulting $r m s, \mathrm{~m} \AA$ & 2.8 & 2.3 & 2.7 & 3.6 \\
Number of lines used in LOP & 58 & 167 & 69 & 34 \\
Wavelength range, $\AA$ & $298-337$ & $337-460$ & $690-930$ & $940-1150$ \\
\hline
\end{tabular}

The rms deviations of all undisturbed identified lines from their Ritz positions, including lines not used in the LOP, are $0.0028 \AA, 0.0023 \AA, 0.0027 \AA$, and $0.0036 \AA$ in the regions of $298-337 \AA, 337-460 \AA, 690-930 \AA$, and 940-1150 $\AA$, respectively. The final estimated uncertainties of wavelength measurements, which were used in the final LOP run, for the $\mathrm{rwmv}_{1}$ and $\mathrm{rwmv}_{2}$ calculations, and in the program LOPT [20], are also included in Table A. These are square root values of combinations of squares of the statistical and systematic 
uncertainties, where the latter were estimated from standard deviations of wavelengths of reference lines from their positions derived from the calibration curves. We have found that the optimized level values given by the IDEN's LOP and those given by the program LOPT are in very good agreement: they differ by a small fraction of calculated level uncertainties.

Comparison of the values of $\mathrm{rwmv}_{1}$ and $\mathrm{rwmv}_{2}$ (see Tables 2 and 3, and Ref. [19]) shows consistency. For the even system having 33 established levels, $\mathrm{rwmv}_{1} \geq \mathrm{rwmv}_{2}$ for all but three levels $(91 \%)$. The remaining three levels have rwmv $_{2}$ values exceeding rwmv values by less than $18 \%$. In the odd system, $51 \%$ of levels have $\mathrm{rwmv}_{1} \geq \mathrm{rwmv}_{2}$. Other $38 \%$ of odd levels have rwmv values exceeding $\mathrm{rwmv}_{1}$ values by less than $50 \%$. We have four levels with the difference $\mathrm{rwmv}_{2}-\mathrm{rwmv}_{1}$ within $50 \%$ to $70 \%$ of the rwmv $\mathrm{ralue}$. One level at $345173.80 \mathrm{~cm}^{-1}(J=11 / 2)$ has $\mathrm{rwmv}_{2}$ value 2.5 times greater than $\mathrm{rwmv}_{1}$ value. According to our validation criteria, the level needs special consideration. It was represented in the LOP by three transitions at $320.7781 \AA, 331.2543 \AA$ and $791.6487 \AA$ with small deviations from the calculated (Ritz) values: $3.9 \mathrm{~m} \AA$, $1.5 \mathrm{~m} \AA$ and $-0.9 \mathrm{~m} \AA$. For the level, the somewhat large value of $\mathrm{rwmv}_{2}$ is due to the high value of the coefficient $A=9.6$ given by Eq. (4) of Ref [19], which, in turn, is explained by the small number of identified transitions (three), with one of them having about 2.4 times greater wavelength and thus about six times greater weight in LOP. Taking these facts into account, we consider the level as identified correctly with acceptable deviations of identified spectral lines from the calculated positions.

As can be seen from the comparison of the values $N, N_{\text {opt }}, \mathrm{rwmv}_{1}$, and $\mathrm{rwmv}_{2}$ (see Tables 2 and 3 ), the most accurately determined level is at $191690.18 \mathrm{~cm}^{-1}(J=7 / 2)$. It is one of the levels with smallest rwmv $\left(0.13 \mathrm{~cm}^{-1}\right)$, with $\mathrm{rwmv}_{1}>\operatorname{rwmv}_{2}\left(0.13 \mathrm{~cm}^{-1}>0.12 \mathrm{~cm}^{-1}\right)$, and with the largest number of higher accuracy $5 d^{2} 6 s-5 d^{2} 6 p$ transitions used in the LOP $\left(N_{\text {opt }}=11\right)$. This level has been chosen to be the "base" in the current presentation of the analysis.

\section{Calculations}

The theoretical description and least squares fitting (LSF) of the configurations is given in the frame of the orthogonal operators approach. Tables 4 and 5 present the parameters obtained (or kept fixed) in the calculations for the even and odd systems. The parameter values were obtained in the final LSF. The ab initio values of the parameters were calculated using the Hartree-Fock method with relativistic correction (HFR) implemented in the code developed by Cowan [21]. For the orthogonal electrostatic parameters, the ab initio (HFR) values were calculated (see Ref. [5]) from the Slater parameters given by the Cowan code. The obtained (or predetermined for fixed parameters) scaling factors (LSF/HFR) are also given in Tables 4 and 5. The values of the fixed parameters $A_{c} \ldots A_{0}$ and $Z_{p p^{\prime}}^{2} \ldots Z_{d^{\prime}}^{3}$ were estimated from the MCDF [23] calculations and the values obtained in the given and neighboring ions.

In both the even and odd parity systems, three configurations were included in the calculations: $\left(5 \mathrm{~d}^{3}+\right.$ $\left.5 d^{2} 6 s+5 d 6 s^{2}\right)$ and $\left(5 d^{2} 6 p+5 d 6 s 6 p+6 s^{2} 6 p\right)$. The interaction between the configurations was added explicitly. The interaction integrals, parameters of unknown configurations and some parameters of the studied configurations were kept fixed at the predetermined values.

As can be seen from Tables 4 and 5, the scaling factors for the strong electrostatic core operators $O_{2}$, $O_{2}{ }^{\prime}$ and spin-orbit interaction parameter $\zeta_{d}$ for the $5 \mathrm{~d}^{3}, 5 \mathrm{~d}^{2} 6 \mathrm{~s}$ and $5 \mathrm{~d}^{2} 6 \mathrm{p}$ configurations are close to each other. These factors, as well as the values of the scaling factor for the electrostatic operator $C_{d s}$, are in agreement with those obtained in Os VI and Ir VII spectra [5,7]. The parameters of the 2-particle magnetic interaction (spinorbit effect) of the $d s$-type $\left(A_{m s o}\right)$ and 3-particle electrostatic $T_{d d s}$ interaction in the $5 \mathrm{~d}^{2} 6 \mathrm{~s}$ configuration are well defined with small uncertainty. These high order parameters are very important as they improve the fit of the $5 \mathrm{~d}^{2} 6 \mathrm{~s}$ configuration considerably. These values are in agreement with those obtained in the neighboring ions in the sequence. For Os VI, Ir VII and Pt VIII, the parameter $A_{m s o}$ has the following values (uncertainties are given in parentheses): $43.5(2.3), 49.9(3.6)$ and 50.1(3.9) $\mathrm{cm}^{-1}$. The values of the parameter $T_{d d s}$ in the sequence are as follows: $-22.3(4.4),-27.3(5.0)$ and $-38.3(6.5) \mathrm{cm}^{-1}$. The trends of the parameters in the $5 \mathrm{~d}^{2} 6 \mathrm{~s}$ configuration are in agreement with those obtained in the $5 \mathrm{~d}^{8} 6 \mathrm{~s}$ and $5 \mathrm{~d}^{7} 6 \mathrm{~s}[13,22]$ configurations. The mean deviations of the fit for the even and odd systems in Pt VIII are $13.8 \mathrm{~cm}^{-1}$ and $116 \mathrm{~cm}^{-1}$, respectively, close to the values in Ir VII [7] $\left(13 \mathrm{~cm}^{-1}\right.$ and $\left.108 \mathrm{~cm}^{-1}\right)$ and in Os VI [5] $\left(10.7 \mathrm{~cm}^{-1}\right.$ and $\left.107 \mathrm{~cm}^{-1}\right)$.

In our calculations presented here, as well as in Ta III, Os VI and Ir VII [2,5,7] analyses, the $5 \mathrm{~d}^{2} 5 \mathrm{f}$ configuration was not included. In the previous work on Pt VIII [1], the interaction between the $5 \mathrm{~d}^{2} 6 \mathrm{p}$ and 
$5 \mathrm{~d}^{2} 5 \mathrm{f}$ configuration was taken into account, which resulted in reduction of the mean deviation of the fit and the uncertainties of the fitted parameters by about $50 \%$.

\section{Acknowledgements}

This work was supported by the National Institute of Standards and Technology, US Department of Commerce (contract number SB1341-12-CQ-0011). The spectra were acquired by Dr. S.S. Churilov of the Institute of Spectroscopy, Troitsk, Moscow, Russian Federation. The initial version of the code for calculations with the orthogonal operators is kindly provided by Dr. P.H.M. Uylings and Dr. A.J.J. Raassen of the van der WaalsZeeman Laboratorium, Universiteit van Amsterdam, The Netherlands, which is greatly appreciated.

\section{References}

[1] R.R. Kildiyarova, A.N. Ryabtsev, S.S. Churilov, V.I. Azarov, Physica Scripta 66 (2002) 51.

[2] V.I. Azarov, W.-U L. Tchang-Brillet, J.-F. Wyart, F.G. Meijer, Physica Scripta 67 (2003) 190.

[3] L. Iglesias, V. Kaufman, O. Garcia-Riquelme, F.R. Rico, Physica Scripta 31 (1985) 173.

[4] R.R. Kildiyarova, Physica Scripta 53 (1996) 668.

[5] A.J.J. Raassen, V.I. Azarov, P.H.M. Uylings, Y.N. Joshi, W.-U L. Tchang-Brillet, A.N. Ryabtsev, Physica Scripta 54 (1996) 56.

[6] R.R. Kildiyarova, S.S. Churilov, Y.N. Joshi, Physica Scripta 58 (1998) 32.

[7] V.I. Azarov, S.S. Churilov, Y.N. Joshi, Physica Scripta 60 (1999) 506.

[8] R.R. Kildiyarova, A.N. Ryabtsev, S.S. Churilov, Opt. Spektrosk. 93(5) (2002) 713-720.

[9] A. Kramida, Yu. Ralchenko, J. Reader and NIST ASD Team, NIST Atomic Spectra Database (version 5.3), 2015, Available: http://physics.nist.gov/asd. National Institute of Standards and Technology, Gaithersburg, MD.

[10] V.I. Azarov, A system for the automatic processing of photospectrograms, Preprint of the Institute of Spectroscopy, Russian Academy of Sciences, Troitsk, 1991, pp. 1 - 32.

[11] V.I. Azarov, R.R. Gayasov, At. Data Nucl. Data Tables 108 (2016) 154-192.

[12] V.I. Azarov, R.R. Gayasov, At. Data Nucl. Data Tables 108 (2016) 118-153.

[13] P.H.M. Uylings, A.J.J. Raassen, J.-F. Wyart, J. Phys. B: At. Mol. Opt. Phys. 26 (1993) 4683.

[14] A.J.J. Raassen, P.H.M. Uylings, Physica Scripta T65 (1996) 84.

[15] P.H.M. Uylings, A.J.J. Raassen, Physica Scripta 54 (1996) 505.

[16] J.E. Hansen, B.R. Judd, A.J.J. Raassen, P.H.M. Uylings, Phys. Rev. Lett. 78 (1997) 3078.

[17] V.I. Azarov, Physica Scripta 44 (1991) 528.

[18] V.I. Azarov, Physica Scripta 48 (1993) 656.

[19] V.I. Azarov, R.R. Gayasov, At. Data Nucl. Data Tables 108 (2016) 81-117.

[20] A.E. Kramida, The program LOPT for least-squares optimization of energy levels, Computer Physics Communications 182 (2011) 419.

[21] R.D. Cowan, The theory of Atomic Structure and Spectra, University of California Press, Berkeley, California, 1981.

Cowan Computer Code as modified by Yu.V. Ralchenko and A.E. Kramida of the Institute of Spectroscopy, Troitsk, Russia. http://das101.troitsk.ru/COWAN

[22] J.-F. Wyart, A.J.J. Raassen, P.H.M. Uylings, Y.N. Joshi, Physica Scripta T47 (1993) 59.

[23] F.A. Parpia, C. Froese Fischer, I.P. Grant, Comput. Phys. Commun. 94 (1996) 249.

\section{Explanation of Tables}

Table 1. Classified lines in the $\left(5 d^{3}+5 d^{2} 6 s\right)-5 d^{2} 6 p$ transition array of Pt VIII

$g A \quad$ Weighted transition probability $\left(10^{8} \mathrm{~s}^{-1} ; g\right.$ is the statistical weight of the upper level)

Int Intensity of the experimental spectral line (arbitrary units)

$\lambda \quad$ Wavelength (in vacuum) of the experimental spectral line $(\AA)$

$v \quad$ Wavenumber of the experimental spectral line $\left(\mathrm{cm}^{-1}\right)$ 


\begin{tabular}{|c|c|c|}
\hline$\lambda_{\text {calc }}$ & \multicolumn{2}{|c|}{ Calculated (Ritz) wavelength $(\AA)$ in vacuum } \\
\hline Unc $\lambda_{\text {calc }}$ & \multicolumn{2}{|c|}{ Uncertainty of the calculated (Ritz) wavelength $(\AA)$} \\
\hline$\Delta(v)$ & \multicolumn{2}{|c|}{ Difference between the experimental and Ritz wavenumbers $\left(\mathrm{cm}^{-1}\right)$} \\
\hline$\Delta(\lambda)$ & \multicolumn{2}{|c|}{ Difference between the experimental and Ritz wavelengths $(\AA)$} \\
\hline$N_{\text {odd }}$ & \multicolumn{2}{|c|}{$\begin{array}{l}\text { Number of observed transitions used to determine the odd level involved } \\
\text { in the transition }\end{array}$} \\
\hline$N_{\text {even }}$ & \multicolumn{2}{|c|}{$\begin{array}{l}\text { Number of observed transitions used to determine the even level involved } \\
\text { in the transition }\end{array}$} \\
\hline Even level & \multicolumn{2}{|c|}{$\begin{array}{l}\text { Experimental energy value }\left(\mathrm{cm}^{-1}\right) \text {, configuration, } L S \text {-term an } J \text {-value of the even } \\
\text { level involved in the transition }\end{array}$} \\
\hline Odd level & \multicolumn{2}{|c|}{$\begin{array}{l}\text { Experimental energy value }\left(\mathrm{cm}^{-1}\right) \text { and } J \text {-value (in parentheses) of the odd } \\
\text { level involved in the transition }\end{array}$} \\
\hline \multirow[t]{10}{*}{ Remark } & \multicolumn{2}{|c|}{ Remarks for the identified transitions: } \\
\hline & $\mathrm{N}$ & New $5 d^{3}-5 d^{2} 6 p$ line added in the present analysis \\
\hline & $\mathrm{m}$ & The Pt VIII line is masked by some other line \\
\hline & 200 & Expected intensity value is 200 \\
\hline & bl & The line is blended by a close line \\
\hline & $*$ & The transition has been excluded from the LOP \\
\hline & $\mathrm{pa}-\mathrm{pd}$ & $\begin{array}{l}\text { Parts of the linelist with assigned wavelength uncertainties } \\
\text { (see text, Table A) }\end{array}$ \\
\hline & Pt VII & $\begin{array}{l}\text { (without "bl") The line is also identified as Pt VII line. The line } \\
\text { does not appear as a blend in the spectrogram, but blending is } \\
\text { possible due to a near coincidence of wavelengths }\end{array}$ \\
\hline & Pt VII (400) & The line is also identified as Pt VII line with expected intensity 400 \\
\hline & $2 \mathrm{~d}$ ord & The line has a contribution from a line seen in the 2 nd order \\
\hline
\end{tabular}

Table 2. Experimental and calculated energy levels $\left(\mathrm{cm}^{-1}\right)$ in the $5 d^{3}$ and $5 d^{2} 6 s$ configurations of Pt VIII

$E_{\text {obs }}$

$E_{\text {calc }}$

$\Delta$

$N$

$N_{\text {opt }}$

rwmv $_{1}$ $\mathrm{rwmV}_{2}$

Unc

Composition
Observed (experimental) energy value $\left(\mathrm{cm}^{-1}\right)$

Calculated from the least squares fitting (LSF) energy value $\left(\mathrm{cm}^{-1}\right)$

Difference between the observed and calculated energy values, $E_{\mathrm{obs}}-E_{\mathrm{calc}}\left(\mathrm{cm}^{-1}\right)$ Number of transitions used to identify the energy level Number of transitions to the level used in the LOP and WMV calculations for the level (see text)

Square root of the weighted mean variance given by Eq. (7) of Ref. [19] $\left(\mathrm{cm}^{-1}\right)$ Square root of the weighted mean variance given by Eq. (8) of Ref. [19] $\left(\mathrm{cm}^{-1}\right)$, except for the levels determined by a single line (see text)

Uncertainty of the level $\left(\mathrm{cm}^{-1}\right)$ with respect to the "base" even level with $J=7 / 2$ at $191690.18 \mathrm{~cm}^{-1}$ (see text)

$L S$-composition of the level. The number preceding the symbol "|" and the term name has the following meaning: 1 stands for $5 \mathrm{~d}^{3}$ and 2 stands for $5 \mathrm{~d}^{2} 6 \mathrm{~s}$.

Table 3. Experimental and calculated energy levels $\left(\mathrm{cm}^{-1}\right)$ in the $5 d^{2} 6 p$ configuration of Pt VIII

$E_{\text {obs }} \quad$ Observed (experimental) energy value $\left(\mathrm{cm}^{-1}\right)$

$E_{\text {calc }} \quad$ Calculated from the least squares fitting (LSF) energy value $\left(\mathrm{cm}^{-1}\right)$

$\Delta \quad$ Difference between the observed and calculated energy values, $E_{\mathrm{obs}}-E_{\text {calc }}\left(\mathrm{cm}^{-1}\right)$

$N \quad$ Number of transitions used to identify the energy level

$N_{\text {opt }} \quad$ Number of transitions from the level used in the LOP and WMV calculations for the level (see text)

rwmv $_{1} \quad$ Square root of the weighted mean variance given by Eq. (7) of Ref. [19] $\left(\mathrm{cm}^{-1}\right)$

$\mathrm{rwmv}_{2}$ Square root of the weighted mean variance given by Eq. (8) of Ref. [19] $\left(\mathrm{cm}^{-1}\right)$, except for the levels determined by a single line (see text)

Unc Uncertainty of the level $\left(\mathrm{cm}^{-1}\right)$ with respect to the "base" even level with $J=7 / 2$ 
$\begin{array}{ll} & \text { at } 191690.18 \mathrm{~cm}^{-1} \text { (see text) } \\ \text { Composition } & L S \text {-composition of the level. }\end{array}$

Table 4. Fitted and calculated parameter values $\left(\mathrm{cm}^{-1}\right)$ in the $5 d^{3}, 5 d^{2} 6 s$ and $5 d 6 s^{2}$ configurations of $P t$ VIII

Parameter Parameter name

Fitted value Parameter value $\left(\mathrm{cm}^{-1}\right)$ obtained in the final least squares fitting (LSF).

Uncertainty of the fit $\left(\mathrm{cm}^{-1}\right)$ is given in parentheses

HFR Ab initio value $\left(\mathrm{cm}^{-1}\right)$ of the parameter. It was calculated using the Hartree-Fock method with relativistic correction (HFR) implemented in the code developed by Cowan [21]

Fitted/HFR Obtained (or predetermined for fixed parameters) scaling factors

Table 5. Fitted and calculated parameter values $\left(\mathrm{cm}^{-1}\right)$ in the $5 d^{2} 6 p, 5 d 6 s 6 p$ and $6 s^{2} 6 p$ configurations of Pt VIII

Parameter

Fitted value

Parameter name

HFR

Parameter value $\left(\mathrm{cm}^{-1}\right)$ obtained in the final least squares fitting (LSF).

Uncertainty of the fit $\left(\mathrm{cm}^{-1}\right)$ is given in parentheses

HFR Ab initio value $\left(\mathrm{cm}^{-1}\right)$ of the parameter. It was calculated using the Hartree-Fock method with relativistic correction (HFR) implemented in the code developed by Cowan [21]

Fitted/HFR Obtained (or predetermined for fixed parameters) scaling factors 
Table 1

Classified lines in the $\left(5 d^{3}+5 d^{2} 6 s\right)-5 d^{2} 6 p$ transition array of Pt VIII.

\begin{tabular}{|c|c|c|c|c|c|c|c|c|c|c|c|c|c|c|}
\hline$\underset{\left(10^{8} \mathrm{~s}^{-1}\right)}{\mathrm{gA}}$ & Int & $\begin{array}{c}\lambda \\
(\AA)\end{array}$ & $\begin{array}{c}v \\
\left(\mathrm{~cm}^{-1}\right)\end{array}$ & $\begin{array}{c}\lambda \text { calc } \\
(\AA)\end{array}$ & $\begin{array}{l}\text { Unc } \lambda \text { calc } \\
(\AA)\end{array}$ & $\begin{array}{l}\Delta(v) \\
\left(\mathrm{cm}^{-1}\right)\end{array}$ & $\begin{array}{l}\Delta(\lambda) \\
(\AA)\end{array}$ & $\mathrm{N}_{\text {odd }}$ & $\mathrm{N}_{\text {even }}$ & $\begin{array}{l}\text { Even level } \\
\left(\mathrm{cm}^{-1}\right)\end{array}$ & & $\begin{array}{l}\text { Odd level } \\
\left(\mathrm{cm}^{-1}\right)\end{array}$ & Remarl & \\
\hline 27 & 28 & 298.5888 & 334908.78 & 298.5877 & 0.0008 & -1.22 & 0.0011 & 12 & 12 & $5 d^{3}{ }^{4} F_{3 / 2}$ & 0.00 & $334910.00(3 / 2)$ & pa 1 & $\mathrm{~N}$ \\
\hline 45 & 23 & 303.5226 & 329464.73 & 303.5225 & 0.0014 & -0.14 & 0.0001 & 4 & 18 & $5 d^{32} \mathrm{D} 2_{5 / 2}$ & 46366.31 & $375831.17(3 / 2)$ & pa 1 & $\mathrm{~N}$ \\
\hline 57 & 55 & 305.8337 & 326975.03 & 305.8332 & 0.0007 & -0.56 & 0.0005 & 9 & 16 & $5 d^{3}{ }^{4} F_{5 / 2}$ & 10194.49 & $337170.08(5 / 2)$ & pa & \\
\hline 58 & 44 & 308.7406 & 323896.49 & 308.7402 & 0.0008 & -0.47 & 0.0004 & 10 & 12 & $5 d^{3}{ }^{4} \mathrm{~F}_{3 / 2}$ & 0.00 & $323896.96(3 / 2)$ & pa 1 & $\mathrm{~N}$ \\
\hline 36 & 46 & 310.8465 & 321702.22 & 310.8483 & 0.0007 & 1.85 & -0.0018 & 10 & 16 & $5 d^{3}{ }^{4} F_{5 / 2}$ & 10194.49 & $331894.85(3 / 2)$ & pa & \\
\hline 44 & 32 & 310.9728 & 321571.57 & 310.9732 & 0.0007 & 0.43 & -0.0004 & 10 & 16 & $5 d^{3}{ }^{4} \mathrm{~F}_{5 / 2}$ & 10194.49 & $331765.63(5 / 2)$ & $\mathrm{pa}$ & \\
\hline 29 & 36 & 311.7060 & 320815.13 & 311.7058 & 0.0007 & -0.16 & 0.0002 & 8 & 17 & $5 d^{3}{ }^{4} P_{5 / 2}$ & 33389.80 & $354205.09(5 / 2)$ & pa 1 & $\mathrm{~N}$ \\
\hline 114 & 138 & 312.4197 & 320082.27 & 312.4158 & 0.0007 & -4.00 & 0.0039 & 9 & 15 & $5 d^{3}{ }^{4} F_{7 / 2}$ & 17083.81 & $337170.08(5 / 2)$ & pa & \\
\hline 121 & 64 & 312.6698 & 319826.19 & 312.6687 & 0.0015 & -1.18 & 0.0012 & 4 & 15 & $5 d^{32} P_{3 / 2}$ & 56003.81 & $375831.17(3 / 2)$ & $\mathrm{pa}$ & \\
\hline 17 & 31 & 313.2131 & 319271.48 & 313.2133 & 0.0013 & 0.24 & -0.0002 & 6 & 17 & $5 d^{3}{ }^{4} P_{5 / 2}$ & 33389.80 & $352661.04(3 / 2)$ & pa & $\mathrm{N}$ \\
\hline 61 & 52 & 316.1439 & 316311.66 & 316.1423 & 0.0007 & -1.61 & 0.0016 & 9 & 16 & $5 d^{32} G_{7 / 2}$ & 31715.83 & $348029.10(7 / 2)$ & pa & \\
\hline 35 & 21 & 316.3087 & 316146.86 & 316.3092 & 0.0007 & 0.54 & -0.0005 & 12 & 14 & $5 d^{3}{ }^{4} P_{3 / 2}$ & 18763.68 & $334910.00(3 / 2)$ & pa & $\mathrm{N}$ \\
\hline 64 & 32 & 316.4025 & 316053.11 & 316.4033 & 0.0007 & 0.75 & -0.0008 & 8 & 16 & $5 d^{32} \mathrm{D} 2_{3 / 2}$ & 38152.74 & $354205.09(5 / 2)$ & pa & \\
\hline 199 & 56 & 316.8147 & 315641.93 & 316.8127 & 0.0007 & -1.98 & 0.0020 & 10 & 16 & $5 \mathrm{~d}^{32} \mathrm{G}_{7 / 2}$ & 31715.83 & $347359.74(5 / 2)$ & $\mathrm{pa}$ & \\
\hline 131 & 51 & 317.3990 & 315060.87 & 317.3957 & 0.0007 & -3.27 & 0.0033 & 11 & 15 & $5 d^{3}{ }^{4} F_{7 / 2}$ & 17083.81 & $332147.94(7 / 2)$ & $\mathrm{pa}$ & \\
\hline 85 & 31 & 317.7841 & 314679.03 & 317.7813 & 0.0008 & -2.78 & 0.0028 & 10 & 15 & $5 d^{3}{ }^{4} \mathrm{~F}_{7 / 2}$ & 17083.81 & $331765.63(5 / 2)$ & $\mathrm{pa}$ & \\
\hline 92 & 104 & 317.8225 & 314641.04 & 317.8243 & 0.0007 & 1.74 & -0.0018 & 9 & 17 & $5 d^{34} P_{5 / 2}$ & 33389.80 & $348029.10(7 / 2)$ & pa & $\mathrm{N}, 40$ \\
\hline 248 & 71 & 317.8649 & 314599.11 & 317.8629 & 0.0008 & -1.96 & 0.0020 & 9 & 12 & $5 d^{32} H_{9 / 2}$ & 33428.03 & $348029.10(7 / 2)$ & pa & \\
\hline 71 & 28 & 318.7692 & 313706.58 & 318.7734 & 0.0008 & 4.10 & -0.0042 & 10 & 16 & $5 d^{3}{ }^{4} F_{5 / 2}$ & 10194.49 & $323896.96(3 / 2)$ & $\mathrm{pa}$ & \\
\hline 49 & 25 & 318.9559 & 313522.96 & 318.9508 & 0.0008 & -4.98 & 0.0051 & 6 & 16 & $5 d^{32} \mathrm{G}_{7 / 2}$ & 31715.83 & $345243.77(7 / 2)$ & pa & $\mathrm{N}$ \\
\hline 118 & 28 & 319.1802 & 313302.59 & 319.1767 & 0.0008 & -3.45 & 0.0035 & 8 & 15 & $5 d^{3}{ }^{4} F_{7 / 2}$ & 17083.81 & $330389.85(9 / 2)$ & pa & \\
\hline 55 & 29 & 319.4884 & 313000.43 & 319.4868 & 0.0007 & -1.51 & 0.0015 & 10 & 14 & $5 d^{3}{ }^{4} \mathrm{P}_{3 / 2}$ & 18763.68 & $331765.63(5 / 2)$ & $\mathrm{pa}$ & \\
\hline 52 & 33 & 319.6871 & 312805.82 & 319.6876 & 0.0014 & 0.49 & -0.0005 & 6 & 9 & $5 d^{32} P_{1 / 2}$ & 39855.71 & $352661.04(3 / 2)$ & $\mathrm{pa}$ & \\
\hline 159 & 46 & 320.7781 & 311741.99 & 320.7742 & 0.0009 & -3.78 & 0.0039 & 3 & 12 & $5 d^{32} \mathrm{H}_{9 / 2}$ & 33428.03 & $345173.80(11 / 2)$ & $\mathrm{pa}$ & \\
\hline 238 & 191 & 320.9426 & 311582.18 & 320.9407 & 0.0008 & -1.82 & 0.0019 & 11 & 11 & $5 d^{3}{ }^{4} F_{9 / 2}$ & 20563.94 & $332147.94(7 / 2)$ & $\mathrm{pa}$ & \\
\hline 75 & 46 & 322.3968 & 310176.81 & 322.3927 & 0.0007 & -3.95 & 0.0041 & 10 & 16 & $5 d^{34} F_{5 / 2}$ & 10194.49 & $320375.24(3 / 2)$ & pa & \\
\hline 45 & 46 & 322.6919 & 309893.16 & 322.6942 & 0.0009 & 2.25 & -0.0023 & 10 & 9 & $5 d^{3}{ }^{4} P_{1 / 2}$ & 22003.95 & $331894.85(3 / 2)$ & pa & \\
\hline 203 & 63 & 322.7653 & 309822.65 & 322.7619 & 0.0009 & -3.27 & 0.0034 & 8 & 11 & $5 d^{34} F_{9 / 2}$ & 20563.94 & $330389.85(9 / 2)$ & pa & \\
\hline 59 & 37 & 323.4084 & 309206.59 & 323.4079 & 0.0007 & -0.42 & 0.0004 & 10 & 16 & $5 d^{32} D 2_{3 / 2}$ & 38152.74 & $347359.74(5 / 2)$ & pa & \\
\hline 153 & 48 & 323.7025 & 308925.62 & 323.6983 & 0.0008 & -4.01 & 0.0042 & 10 & 16 & $5 d^{34} F_{5 / 2}$ & 10194.49 & $319124.12(7 / 2)$ & pa & \\
\hline 49 & 41 & 324.1178 & 308529.76 & 324.1115 & 0.0008 & -6.00 & 0.0063 & 7 & 16 & $5 \mathrm{~d}^{32} \mathrm{G}_{7 / 2}$ & 31715.83 & $340251.58(9 / 2)$ & pa * & \\
\hline 187 & 35 & 324.8476 & 307836.64 & 324.8454 & 0.0007 & -2.14 & 0.0023 & 8 & 18 & $5 d^{32} D 2_{5 / 2}$ & 46366.31 & $354205.09(5 / 2)$ & pa & \\
\hline 143 & 48 & 325.9198 & 306823.97 & 325.9202 & 0.0008 & 0.42 & -0.0004 & 7 & 12 & $5 d^{32} \mathrm{H}_{9 / 2}$ & 33428.03 & $340251.58(9 / 2)$ & pa & \\
\hline 182 & 68 & 326.0973 & 306656.94 & 326.0942 & 0.0010 & -2.92 & 0.0031 & 5 & 12 & $5 d^{3}{ }^{4} F_{3 / 2}$ & 0.00 & $306659.86(1 / 2)$ & pa & \\
\hline 345 & 159 & 327.0985 & 305718.27 & 327.0950 & 0.0009 & -3.33 & 0.0036 & 5 & 11 & $5 d^{3}{ }^{4} F_{9 / 2}$ & 20563.94 & $326285.54(11 / 2)$ & pa & \\
\hline
\end{tabular}




\begin{tabular}{|c|c|c|c|c|c|c|c|c|c|c|}
\hline 41 & 27 & 327.3189 & 305512.47 & 327.3133 & 0.0009 & -5.23 & 0.0056 & 9 & 16 & $5 d^{3}{ }^{4} F_{5 / 2}$ \\
\hline 86 & 22 & 328.9283 & 304017.62 & 328.9206 & 0.0009 & -7.11 & 0.0077 & 13 & 12 & $5 d^{3}{ }^{4} \mathrm{~F}_{3 / 2}$ \\
\hline 271 & 72 & 329.4055 & 303577.16 & 329.4016 & 0.0008 & -3.63 & 0.0039 & 8 & 16 & $5 d^{32} F_{5 / 2}$ \\
\hline 115 & 45 & 329.4540 & 303532.51 & 329.4514 & 0.0009 & -2.44 & 0.0026 & 7 & 11 & $5 d^{3}{ }^{4} F_{9 / 2}$ \\
\hline 146 & 62 & 330.8693 & 302234.16 & 330.8687 & 0.0008 & -0.58 & 0.0006 & 9 & 15 & \\
\hline 370 & 161 & 331.0830 & 302039.03 & 331.0816 & 0.0008 & -1.28 & 0.0014 & 10 & 15 & \\
\hline 131 & 161 & 331.0830 & 302039.03 & 331.0855 & 0.0014 & 2.28 & -0.0025 & 6 & 16 & \\
\hline 1693 & 784 & 331.2543 & 301882.85 & 331.2528 & 0.0011 & -1.38 & 0.0015 & 3 & 7 & $5 \mathrm{~d}^{32} \mathrm{H}_{11 / 2}$ \\
\hline 66 & 14 & 331.6591 & 301514.44 & 331.6527 & 0.0007 & -5.77 & 0.0063 & 12 & 17 & \\
\hline 229 & 66 & 331.9886 & 301215.16 & 331.9881 & 0.0008 & -0.44 & 0.0005 & 6 & 16 & $\mathrm{D} 2_{3 / 2}$ \\
\hline 184 & 32 & 332.2339 & 300992.77 & 332.2332 & 0.0007 & -0.66 & 0.0007 & 10 & 18 & $5 d^{3}{ }^{2} D 2_{5 / 2}$ \\
\hline 88 & 24 & 332.7174 & 300555.36 & 332.7180 & 0.0008 & 0.49 & -0.0005 & 9 & 14 & \\
\hline 40 & 12 & 332.8540 & 300432.05 & 332.8539 & 0.0008 & -0.06 & 0.0001 & 11 & 16 & $\mathrm{G}_{7 / 2}$ \\
\hline 46 & 35 & 333.2985 & 300031.35 & 333.2966 & 0.0010 & -1.68 & 0.0019 & 11 & 12 & \\
\hline 125 & 16 & 333.5137 & 299837.79 & 333.5134 & 0.0017 & -0.23 & 0.0003 & 4 & 10 & $5 d^{32} D 1_{3 / 2}$ \\
\hline 117 & 21 & 333.8803 & 299508.57 & 333.8757 & 0.0011 & -4.06 & 0.0045 & 6 & 9 & \\
\hline 84 & 19 & 334.4275 & 299018.48 & 334.4288 & 0.0008 & 1.13 & -0.0013 & 9 & 16 & $\mathrm{D} 2_{3 / 2}$ \\
\hline 82 & 27 & 334.5844 & 298878.29 & 334.5853 & 0.0007 & 0.83 & -0.0009 & 6 & 18 & $5 \mathrm{~d}^{3}{ }^{2} \mathrm{D} 2_{5 / 2}$ \\
\hline 383 & 118 & 334.7188 & 298758.22 & 334.7189 & 0.0008 & 0.08 & -0.0001 & 11 & 17 & $5 d^{3}{ }^{4} P_{5 / 2}$ \\
\hline 44 & 296 & 335.1471 & 298376.40 & 335.1529 & 0.0010 & 5.10 & -0.0057 & 10 & 9 & \\
\hline 765 & 296 & 335.1471 & 298376.40 & 335.1478 & 0.0008 & 0.58 & -0.0006 & 10 & 17 & $5 d^{3}{ }^{4} P_{5 / 2}$ \\
\hline 732 & 290 & 335.6580 & 297922.26 & 335.6613 & 0.0009 & 2.88 & -0.0032 & 7 & 11 & \\
\hline 187 & 53 & 335.7226 & 297864.96 & 335.7205 & 0.0008 & -1.90 & 0.0021 & 10 & 16 & $5 d^{32} G_{7 / 2}$ \\
\hline 56 & 20 & 335.7907 & 297804.58 & 335.7937 & 0.0025 & 2.65 & -0.0030 & 2 & 15 & \\
\hline 907 & 128 & 336.7150 & 296987.10 & 336.7163 & 0.0018 & 1.19 & -0.0014 & 4 & 7 & $5 d^{32} D 1_{5 / 2}$ \\
\hline 410 & 152 & 336.7408 & 296964.33 & 336.7436 & 0.0009 & 2.51 & -0.0028 & 8 & 12 & $5 d^{3}{ }^{2} \mathrm{H}_{9 / 2}$ \\
\hline 75 & 152 & 336.7408 & 296964.33 & 336.7434 & 0.0011 & 2.32 & -0.0026 & 7 & 7 & $5 \mathrm{~d}^{32} \mathrm{H}_{11 / 2}$ \\
\hline 354 & 106 & 336.9978 & 296737.80 & 337.0005 & 0.0007 & 2.37 & -0.0027 & 10 & 16 & $5 d^{3}{ }^{2} F_{5 / 2}$ \\
\hline 617 & 78 & 337.0898 & 296656.87 & 337.0894 & 0.0015 & -0.37 & 0.0004 & 6 & 15 & \\
\hline 38 & 322 & 337.6731 & 296144.41 & 337.6626 & 0.0010 & -9.21 & 0.0105 & 13 & 12 & $5 d^{3}{ }^{4} F_{3 / 2}$ \\
\hline 156 & 22 & 337.7698 & 296059.62 & 337.7709 & 0.0009 & 0.98 & -0.0011 & 9 & 9 & $5 d^{3}{ }^{2} G_{9 / 2}$ \\
\hline 93 & 87 & 338.1113 & 295760.60 & 338.1149 & 0.0010 & 3.11 & -0.0036 & 7 & 12 & $5 d^{3}{ }^{4} F_{3 / 2}$ \\
\hline 680 & 343 & 338.4697 & 295447.42 & 338.4686 & 0.0008 & -0.92 & 0.0011 & 9 & 14 & \\
\hline 51 & 58 & 338.9016 & 295070.90 & 338.9025 & 0.0008 & 0.77 & -0.0009 & 10 & 15 & $5 d^{3}{ }^{4} F_{7 / 2}$ \\
\hline 193 & 86 & 339.2372 & 294778.99 & 339.2372 & 0.0008 & 0.02 & 0.0000 & 10 & 14 & $5 d^{32} F_{7 / 2}$ \\
\hline 37 & 57 & 339.7106 & 294368.21 & 339.7109 & 0.0010 & 0.30 & -0.0003 & 9 & 12 & $5 d^{3}{ }^{4} F_{3 / 2}$ \\
\hline 62 & 49 & 339.7382 & 294344.29 & 339.7404 & 0.0008 & 1.87 & -0.0022 & 12 & 15 & \\
\hline 213 & 142 & 340.3322 & 293830.56 & 340.3326 & 0.0008 & 0.31 & -0.0004 & 13 & 16 & \\
\hline 4 & 34 & 340.5895 & 293608.58 & 340.5845 & 0.0008 & -4.31 & 0.0050 & 10 & 16 & \\
\hline 4194 & 780 & 340.9816 & 293270.96 & 340.9789 & 0.0010 & -2.36 & 0.0027 & 6 & 9 & $5 \mathrm{~d} \quad \mathrm{G}_{9 / 2}$ \\
\hline 132 & 109 & 341.4647 & 292856.04 & 341.4630 & 0.0010 & -1.47 & 0.0017 & 5 & 12 & $5 d^{3}{ }^{3} H_{9 / 2}$ \\
\hline
\end{tabular}

$10194.49315712 .19(5 / 2) \quad$ pa $*$ $0.00304024 .73(5 / 2)$ pa $* \mathrm{~N}$ $50624.30 \quad 354205.09(5 / 2)$ pa $20563.94324098 .89(7 / 2)$ pa $17083.81319318 .55(5 / 2)$ pa $17083.81319124 .12(7 / 2)$ pa $50624.30 \quad 352661.04(3 / 2)$ pa $43289.57345173 .80(11 / 2)$ pa $33389.80334910 .00(3 / 2)$ pa $* \mathrm{~N}$ $38152.74339368 .34(1 / 2)$ pa $46366.31347359 .74(5 / 2)$ pa $18763.68319318 .55(5 / 2)$ pa $31715.83332147 .94(7 / 2)$ pa $\mathrm{N}$ $0.00300033 .03(3 / 2)$ pa N, Pt IX $75993.15375831 .17(3 / 2)$ pa $\mathrm{N}$ $39855.71339368 .34(1 / 2)$ pa $38152.74337170 .08(5 / 2)$ pa $\mathrm{N}$ $46366.31345243 .77(7 / 2)$ pa $33389.80 \quad 332147.94(7 / 2)$ pa $22003.95320375 .24(3 / 2)$ pa $* \mathrm{~N}, 40, \mathrm{~m}$ $33389.80 \quad 331765.63(5 / 2)$ pa $20563.94318483 .31(9 / 2)$ pa $31715.83 \quad 329582.69(5 / 2)$ pa $56003.81353805 .73(1 / 2)$ pa $78845.26375831 .17(3 / 2)$ pa $\mathrm{N}$ $33428.03 \quad 330389.85(9 / 2)$ pa $43289.57340251 .58(9 / 2)$ pa $*$ N, 45 $50624.30347359 .74(5 / 2)$ pa $56003.81352661 .04(3 / 2)$ pa

$0.00296153 .62(5 / 2) \quad \mathrm{pb} * \mathrm{~N}, 150$, Pt IX $51970.45348029 .10(7 / 2) \quad \mathrm{pb}$ $0.00295757 .49(1 / 2) \quad \mathrm{pb}$ $52580.76348029 .10(7 / 2) \quad \mathrm{pb}$ $17083.81312153 .94(9 / 2) \quad p b \quad \mathrm{~N}$ $52580.76347359 .74(5 / 2) \quad$ pb $0.00 \quad 294367.91(3 / 2) \quad \mathrm{pb} \quad \mathrm{N}$ $\begin{array}{llll}17083.81 & 311426.23(7 / 2) & \text { pb } \mathrm{N}\end{array}$ $10194.49304024 .73(5 / 2) \mathrm{pb}$ $38152.74331765 .63(5 / 2) \quad \mathrm{pb} * \mathrm{~N}$ $51970.45345243 .77(7 / 2) \quad \mathrm{pb}$ $33428.03 \quad 326285.54(11 / 2) \mathrm{pb}$ 


\begin{tabular}{|c|c|c|c|c|c|c|c|c|c|c|}
\hline 800 & 514 & 341.9631 & 292429.21 & 341.9617 & 0.0009 & -1.22 & 0.0014 & 5 & 16 & $5 d^{3}{ }^{4} F_{5 / 2}$ \\
\hline 287 & 274 & 342.9489 & 291588.63 & 342.9473 & 0.0009 & -1.37 & 0.0016 & 10 & 11 & $5 d^{3}{ }^{4} F_{9 / 2}$ \\
\hline 76 & 75 & 343.1328 & 291432.35 & 343.1356 & 0.0008 & 2.40 & -0.0028 & 10 & 16 & $5 d^{32} D 2_{3 / 2}$ \\
\hline 141 & 92 & 343.2236 & 291355.26 & 343.2228 & 0.0009 & -0.68 & 0.0008 & 10 & 15 & $5 d^{3}{ }^{2} P_{3 / 2}$ \\
\hline 284 & 178 & 343.8067 & 290861.11 & 343.8053 & 0.0009 & -1.18 & 0.0014 & 12 & 11 & $5 d^{3}{ }^{4} F_{9 / 2}$ \\
\hline 2658 & 854 & 344.0305 & 290671.90 & 344.0317 & 0.0009 & 1.05 & -0.0012 & 7 & 12 & $5 \mathrm{~d}^{3}{ }^{2} \mathrm{H}_{9 / 2}$ \\
\hline 902 & 511 & 344.2271 & 290505.89 & 344.2256 & 0.0008 & -1.27 & 0.0015 & 10 & 17 & \\
\hline 270 & 283 & 345.0194 & 289838.77 & 345.0197 & 0.0009 & 0.23 & -0.0003 & 11 & 16 & $5 d^{3}{ }^{4} F_{5 / 2}$ \\
\hline 404 & 330 & 346.5098 & 288592.13 & 346.5118 & 0.0008 & 1.67 & -0.0020 & 8 & 15 & \\
\hline 113 & 101 & 346.5630 & 288547.83 & 346.5680 & 0.0007 & 4.13 & -0.0050 & 12 & 18 & $5 d^{3}{ }^{2} \mathrm{D} 2_{5 / 2}$ \\
\hline 2409 & 882 & 346.8842 & 288280.64 & 346.8836 & 0.0010 & -0.49 & 0.0006 & 7 & 9 & $5 d^{32} G_{9 / 2}$ \\
\hline 134 & 137 & 347.3438 & 287899.19 & 347.3474 & 0.0010 & 3.01 & -0.0036 & 5 & 14 & $5 d^{3}{ }^{4} P_{3 / 2}$ \\
\hline 242 & 166 & 347.5466 & 287731.20 & 347.5460 & 0.0008 & -0.52 & 0.0006 & 4 & 16 & $5 d^{32} D 2_{3 / 2}$ \\
\hline 226 & 105 & 347.6182 & 287671.93 & 347.6195 & 0.0008 & 1.11 & -0.0013 & 7 & 14 & \\
\hline 2235 & 926 & 347.7009 & 287603.51 & 347.7019 & 0.0009 & 0.80 & -0.0010 & 9 & 16 & $5 d^{32} G_{7 / 2}$ \\
\hline 57 & 141 & 347.9000 & 287438.92 & 347.9013 & 0.0009 & 1.09 & -0.0013 & 10 & 16 & \\
\hline 664 & 423 & 347.9360 & 287409.18 & 347.9371 & 0.0009 & 0.89 & -0.0011 & 10 & 16 & $5 d^{32} G_{7 / 2}$ \\
\hline 532 & 684 & 348.1698 & 287216.18 & 348.1726 & 0.0011 & 2.28 & -0.0028 & 4 & 12 & $5 d^{34} F_{3 / 2}$ \\
\hline 5194 & 1086 & 348.3111 & 287099.66 & 348.3103 & 0.0012 & -0.62 & 0.0008 & 8 & 7 & $5 d^{32} \mathrm{H}_{11 / 2}$ \\
\hline 220 & 183 & 348.4462 & 286988.35 & 348.4497 & 0.0008 & 2.90 & -0.0035 & 10 & 17 & $5 d^{3}{ }^{4} P_{5 / 2}$ \\
\hline 1900 & 914 & 348.5037 & 286941.00 & 348.5038 & 0.0009 & 0.08 & -0.0001 & 13 & 15 & $5 d^{34} F_{7 / 2}$ \\
\hline 82 & 123 & 349.0761 & 286470.49 & 349.0748 & 0.0011 & -1.04 & 0.0013 & 8 & 12 & $5 d^{34} F_{3 / 2}$ \\
\hline 400 & 255 & 349.6156 & 286028.43 & 349.6152 & 0.0012 & -0.32 & 0.0004 & 4 & 9 & $5 d^{32} \mathrm{P}_{1 / 2}$ \\
\hline 571 & 651 & 349.7024 & 285957.43 & 349.7003 & 0.0008 & -1.70 & 0.0021 & 13 & 16 & $5 d^{34} F_{5 / 2}$ \\
\hline 242 & 125 & 349.9222 & 285777.81 & 349.9175 & 0.0007 & -3.82 & 0.0047 & 11 & 18 & $5 d^{32} D 2_{5 / 2}$ \\
\hline 172 & 161 & 349.9602 & 285746.78 & 349.9633 & 0.0008 & 2.55 & -0.0031 & 10 & 16 & $5 d^{32} D 2_{3 / 2}$ \\
\hline 112 & 190 & 349.9774 & 285732.74 & 349.9755 & 0.0009 & -1.58 & 0.0019 & 10 & 17 & $5 d^{34} P_{5 / 2}$ \\
\hline 1130 & 643 & 350.0238 & 285694.86 & 350.0223 & 0.0010 & -1.23 & 0.0015 & 10 & 12 & $5 d^{32} H_{9 / 2}$ \\
\hline 1062 & 515 & 350.2279 & 285528.37 & 350.2277 & 0.0008 & -0.18 & 0.0002 & 10 & 18 & $5 d^{32} D 2_{5 / 2}$ \\
\hline 75 & 37 & 350.3860 & 285399.53 & 350.3863 & 0.0008 & 0.21 & -0.0003 & 10 & 18 & $5 d^{32} D 2_{5 / 2}$ \\
\hline 139 & 100 & 350.5566 & 285260.64 & 350.5561 & 0.0008 & -0.41 & 0.0005 & 13 & 14 & $5 d^{34} P_{3 / 2}$ \\
\hline 2498 & 1138 & 350.7398 & 285111.64 & 350.7414 & 0.0010 & 1.32 & -0.0016 & 8 & 11 & $5 d^{34} F_{9 / 2}$ \\
\hline 1244 & 721 & 350.8094 & 285055.08 & 350.8091 & 0.0009 & -0.21 & 0.0003 & 7 & 12 & $5 d^{32} \mathrm{H}_{9 / 2}$ \\
\hline 362 & 368 & 351.3040 & 284653.75 & 351.3013 & 0.0011 & -2.17 & 0.0027 & 5 & 9 & $5 d^{34} P_{1 / 2}$ \\
\hline 1621 & 648 & 351.3861 & 284587.24 & 351.3835 & 0.0009 & -2.08 & 0.0026 & 9 & 14 & $5 d^{32} F_{7 / 2}$ \\
\hline 1031 & 453 & 351.7603 & 284284.50 & 351.7588 & 0.0008 & -1.20 & 0.0015 & 12 & 16 & $5 d^{32} F_{5 / 2}$ \\
\hline 495 & 549 & 351.8985 & 284172.85 & 351.8978 & 0.0009 & -0.57 & 0.0007 & 9 & 16 & $5 d^{34} F_{5 / 2}$ \\
\hline 105 & 138 & 352.0590 & 284043.30 & 352.0615 & 0.0012 & 2.05 & -0.0025 & 10 & 9 & $5 d^{32} P_{1 / 2}$ \\
\hline 322 & 330 & 352.1180 & 283995.71 & 352.1172 & 0.0010 & -0.66 & 0.0008 & 9 & 16 & $5 d^{32} G_{7 / 2}$ \\
\hline 405 & 358 & 352.2875 & 283859.06 & 352.2848 & 0.0009 & -2.17 & 0.0027 & 5 & 14 & $5 d^{3{ }^{4}} P_{3 / 2}$ \\
\hline 2 & 41 & 352.9040 & 283363.18 & 352.9023 & 0.0010 & -1.35 & 0.0017 & 6 & 15 & $5 d^{32} P_{3 / 2}$ \\
\hline
\end{tabular}

$10194.49302624 .91(3 / 2) \quad \mathrm{pb}$ $20563.94312153 .94(9 / 2) \quad \mathrm{pb}$ $38152.74329582 .69(5 / 2) \quad$ pb $\quad \mathrm{N}$ $56003.81347359 .74(5 / 2) \quad \mathrm{pb}$ $20563.94311426 .23(7 / 2) \quad \mathrm{pb}$ $33428.03 \quad 324098.89(7 / 2) \quad \mathrm{pb}$ $33389.80 \quad 323896.96(3 / 2)$ pb Pt IX(250) $10194.49300033 .03(3 / 2) \quad \mathrm{pb}$ $17083.81305674 .26(7 / 2) \quad \mathrm{pb}$ $46366.31334910 .00(3 / 2) \quad \mathrm{pb}$ $51970.45340251 .58(9 / 2) \quad \mathrm{pb}$ $18763.68306659 .86(1 / 2) \quad \mathrm{pb}$ $38152.74325884 .46(1 / 2) \quad \mathrm{pb}$ $52580.76340251 .58(9 / 2) \quad \mathrm{pb}$ $31715.83 \quad 319318.55(5 / 2) \quad \mathrm{pb}$ $10194.49297632 .31(7 / 2) \quad \mathrm{pb}$ $31715.83 \quad 319124.12(7 / 2) \quad \mathrm{pb}$ $0.00 \quad 287213.90(1 / 2) \quad \mathrm{pb}$ $43289.57330389 .85(9 / 2) \quad \mathrm{pb}$ $33389.80 \quad 320375.24(3 / 2) \quad p b$ $17083.81304024 .73(5 / 2) \quad \mathrm{pb}$ $0.00286471 .53(5 / 2) \quad \mathrm{pb}$ $39855.71325884 .46(1 / 2) \quad \mathrm{pb}$ $10194.49296153 .62(5 / 2) \quad \mathrm{pb}$ $\begin{array}{llll}46366.31 & 332147.94(7 / 2) & \text { pb } & \mathrm{N}\end{array}$ $38152.74323896 .96(3 / 2) \quad$ pb $33389.80 \quad 319124.12(7 / 2) \quad$ pb $\mathrm{N}$ $33428.03 \quad 319124.12(7 / 2) \quad \mathrm{pb}$ $46366.31 \quad 331894.85(3 / 2) \quad \mathrm{pb}$ $46366.31331765 .63(5 / 2)$ pb $\mathrm{N}$ $18763.68304024 .73(5 / 2)$ pb $\mathrm{N}$ $20563.94305674 .26(7 / 2) \quad \mathrm{pb}$ $33428.03 \quad 318483.31(9 / 2) \quad \mathrm{pb}$ $22003.95306659 .86(1 / 2) \quad \mathrm{pb}$ $52580.76337170 .08(5 / 2) \quad \mathrm{pb}$ $50624.30 \quad 334910.00(3 / 2) \quad \mathrm{pb}$ $10194.49294367 .91(3 / 2) \quad \mathrm{pb}$ $\begin{array}{lll}39855.71 & 323896.96(3 / 2) & \mathrm{pb}\end{array}$ $31715.83 \quad 315712.19(5 / 2) \quad \mathrm{pb}$ $18763.68302624 .91(3 / 2) \quad \mathrm{pb}$ $56003.81339368 .34(1 / 2) \quad$ pb $* \mathrm{~N}$ 


\begin{tabular}{|c|c|c|c|c|c|c|c|c|c|c|}
\hline 1021 & 498 & 353.0873 & 283216.08 & 353.0869 & 0.0007 & -0.30 & 0.0004 & 10 & 18 & $5 d^{32} D 2_{5 / 2}$ \\
\hline 274 & 385 & 353.3610 & 282996.71 & 353.3619 & 0.0013 & 0.73 & -0.0009 & 5 & 7 & $5 d^{32} \mathrm{H}_{11 / 2}$ \\
\hline 544 & 424 & 354.3298 & 282222.95 & 354.3303 & 0.0008 & 0.44 & -0.0005 & 10 & 16 & $5 d^{32} D 2_{3 / 2}$ \\
\hline 95 & 82 & 355.0651 & 281638.49 & 355.0676 & 0.0010 & 2.01 & -0.0025 & 6 & 17 & $5 d^{34} P_{5 / 2}$ \\
\hline 617 & 737 & 355.5330 & 281267.84 & 355.5311 & 0.0009 & -1.50 & 0.0019 & 11 & 14 & \\
\hline 712 & 737 & 355.5330 & 281267.84 & 355.5296 & 0.0008 & -2.71 & 0.0034 & 10 & 16 & $5 d^{32} F_{5 / 2}$ \\
\hline 288 & 401 & 355.6607 & 281166.85 & 355.6620 & 0.0009 & 1.04 & -0.0013 & 9 & 16 & $5 d^{32} \mathrm{D} 2_{3 / 2}$ \\
\hline 498 & 401 & 355.6607 & 281166.85 & 355.6614 & 0.0010 & 0.58 & -0.0007 & 9 & 15 & $5 d^{32} P_{3 / 2}$ \\
\hline 200 & 241 & 356.3548 & 280619.20 & 356.3526 & 0.0011 & -1.77 & 0.0022 & 5 & 9 & $5 d^{34} P_{1 / 2}$ \\
\hline 1298 & 912 & 356.4469 & 280546.70 & 356.4446 & 0.0009 & -1.81 & 0.0023 & 10 & 15 & $5 d^{34} F_{7 / 2}$ \\
\hline 218 & 488 & 356.4815 & 280519.47 & 356.4814 & 0.0012 & -0.07 & 0.0001 & 10 & 9 & $5 d^{32} P_{1 / 2}$ \\
\hline 273 & 293 & 356.5837 & 280439.07 & 356.5849 & 0.0009 & 0.96 & -0.0012 & 10 & 16 & $5 d^{32} G_{7 / 2}$ \\
\hline 725 & 421 & 356.8577 & 280223.74 & 356.8552 & 0.0010 & -1.96 & 0.0025 & 4 & 15 & $5 d^{32} P_{3 / 2}$ \\
\hline 341 & 373 & 356.9131 & 280180.25 & 356.9166 & 0.0010 & 2.75 & -0.0035 & 11 & 9 & $5 d^{32} G_{9 / 2}$ \\
\hline 1173 & 786 & 357.5126 & 279710.42 & 357.5126 & 0.0009 & 0.02 & 0.0000 & 12 & 16 & $5 d^{32} G_{7 / 2}$ \\
\hline 1088 & 692 & 357.6959 & 279567.09 & 357.6958 & 0.0009 & -0.10 & 0.0001 & 11 & 14 & $5 d^{32} F_{7 / 2}$ \\
\hline 533 & 613 & 358.3321 & 279070.73 & 358.3333 & 0.0009 & 0.92 & -0.0012 & 13 & 15 & $5 d^{34} F_{7 / 2}$ \\
\hline 132 & 98 & 358.4735 & 278960.65 & 358.4764 & 0.0008 & 2.26 & -0.0029 & 10 & 16 & $5 d^{32} F_{5 / 2}$ \\
\hline 192 & 150 & 358.5447 & 278905.25 & 358.5435 & 0.0010 & -0.94 & 0.0012 & 12 & 15 & $5 d^{32} P_{3 / 2}$ \\
\hline 570 & 616 & 358.7749 & 278726.30 & 358.7754 & 0.0009 & 0.39 & -0.0005 & 10 & 12 & $5 d^{32} H_{9 / 2}$ \\
\hline 143 & 301 & 359.1733 & 278417.13 & 359.1704 & 0.0010 & -2.27 & 0.0029 & 8 & 9 & $5 d^{32} G_{9 / 2}$ \\
\hline 412 & 651 & 359.2052 & 278392.41 & 359.2045 & 0.0009 & -0.54 & 0.0007 & 7 & 15 & $5 d^{34} F_{7 / 2}$ \\
\hline 263 & 111 & 359.4388 & 278211.48 & 359.4382 & 0.0011 & -0.46 & 0.0006 & 8 & 10 & $5 d^{32} \mathrm{D}_{3 / 2}$ \\
\hline 226 & 264 & 359.6703 & 278032.41 & 359.6746 & 0.0011 & 3.33 & -0.0043 & 11 & 9 & $5 d^{34} P_{1 / 2}$ \\
\hline 550 & 544 & 359.7103 & 278001.49 & 359.7146 & 0.0010 & 3.29 & -0.0043 & 12 & 12 & $5 d^{32} H_{9 / 2}$ \\
\hline 248 & 425 & 359.9575 & 277810.58 & 359.9594 & 0.0009 & 1.48 & -0.0019 & 8 & 14 & \\
\hline 877 & 425 & 359.9575 & 277810.58 & 359.9549 & 0.0031 & -2.01 & 0.0026 & 2 & 10 & $5 d^{32} D_{1 / 2}$ \\
\hline 301 & 258 & 360.0549 & 277735.42 & 360.0586 & 0.0008 & 2.85 & -0.0037 & 7 & 18 & $5 d^{32} \mathrm{D} 2_{5 / 2}$ \\
\hline 235 & 377 & 360.2818 & 277560.51 & 360.2832 & 0.0010 & 1.05 & -0.0014 & 9 & 16 & $5 d^{32} D 2_{3 / 2}$ \\
\hline 39 & 118 & 360.5054 & 277388.35 & 360.5033 & 0.0009 & -1.58 & 0.0021 & 13 & 14 & $5 d^{34} P_{3 / 2}$ \\
\hline 771 & 902 & 360.9194 & 277070.17 & 360.9217 & 0.0010 & 1.80 & -0.0023 & 10 & 11 & $5 d^{34} F_{9 / 2}$ \\
\hline 518 & 300 & 361.0079 & 277002.25 & 361.0083 & 0.0009 & 0.32 & -0.0004 & 10 & 14 & $5 d^{32} F_{7 / 2}$ \\
\hline 341 & 866 & 361.0207 & 276992.43 & 361.0189 & 0.0009 & -1.38 & 0.0018 & 7 & 14 & $5 d^{34} P_{3 / 2}$ \\
\hline 707 & 579 & 361.1765 & 276872.94 & 361.1757 & 0.0010 & -0.60 & 0.0008 & 6 & 16 & $5 d^{32} \mathrm{D} 2_{3 / 2}$ \\
\hline 356 & 231 & 361.4452 & 276667.11 & 361.4442 & 0.0017 & -0.78 & 0.0010 & 6 & 10 & $5 d^{32} D 1_{3 / 2}$ \\
\hline 1008 & 1025 & 361.9568 & 276276.06 & 361.9555 & 0.0009 & -0.98 & 0.0013 & 8 & 16 & $5 d^{34} F_{5 / 2}$ \\
\hline 78 & 58 & 362.4645 & 275889.09 & 362.4619 & 0.0010 & -1.96 & 0.0026 & 10 & 15 & \\
\hline 20 & 38 & 362.6314 & 275762.11 & 362.6318 & 0.0010 & 0.29 & -0.0004 & 10 & 15 & $5 d^{32} P_{2}$ \\
\hline 135 & 248 & 362.8417 & 275602.28 & 362.8391 & 0.0009 & -1.95 & 0.0026 & 9 & 14 & $5 d^{34} P_{3 / 2}$ \\
\hline 1043 & 519 & 363.1616 & 275359.51 & 363.1612 & 0.0012 & -0.32 & 0.0004 & 8 & 7 & $5 d^{32} \mathrm{D} 1$ \\
\hline 900 & 768 & 363.3829 & 275191.82 & 363.3804 & 0.0013 & -1.93 & 0.0025 & 7 & 7 & $5 d^{32} H_{11 / 2}$ \\
\hline
\end{tabular}

$46366.31329582 .69(5 / 2) \quad \mathrm{pb}$ $43289.57326285 .54(11 / 2) \mathrm{pb}$ $38152.74320375 .24(3 / 2) \quad p b$ $33389.80 \quad 315026.28(3 / 2) \quad \mathrm{pb}$ $18763.68300033 .03(3 / 2) \quad \mathrm{pb}$ $50624.30331894 .85(3 / 2)$ pb 400 $38152.74319318 .55(5 / 2) \quad \mathrm{pb}$ $56003.81337170 .08(5 / 2) \quad \mathrm{pb}$ $22003.95302624 .91(3 / 2) \quad \mathrm{pb}$ $17083.81297632 .31(7 / 2) \quad \mathrm{pb}$ $39855.71320375 .24(3 / 2)$ pb N, 300 $31715.83 \quad 312153.94(9 / 2) \quad \mathrm{pb}$ $56003.81336229 .51(1 / 2) \quad \mathrm{pb}$ $51970.45 \quad 332147.94(7 / 2) \quad$ pb $31715.83 \quad 311426.23(7 / 2) \quad \mathrm{pb}$ $\begin{array}{lll}52580.76 & 332147.94(7 / 2) & \text { pb }\end{array}$ $17083.81296153 .62(5 / 2) \quad \mathrm{pb}$ $50624.30 \quad 329582.69(5 / 2) \quad \mathrm{pb}$ $56003.81334910 .00(3 / 2) \quad$ pb $33428.03 \quad 312153.94(9 / 2) \quad \mathrm{pb}$ $51970.45330389 .85(9 / 2)$ pb $\mathrm{N}$ $17083.81 \quad 295476.76(9 / 2) \quad \mathrm{pb}$ $75993.15354205 .09(5 / 2) \quad \mathrm{pb}$ $22003.95300033 .03(3 / 2) \quad \mathrm{pb}$ $33428.03 \quad 311426.23(7 / 2) \quad \mathrm{pb}$ $52580.76330389 .85(9 / 2)$ pb $\mathrm{N}$ $75993.15353805 .73(1 / 2) \quad \mathrm{pb}$ $\begin{array}{lll}46366.31 & 324098.89(7 / 2) & \mathrm{pb}\end{array}$ $38152.74315712 .19(5 / 2) \quad \mathrm{pb}$ $18763.68296153 .62(5 / 2) \quad \mathrm{pb}$ $20563.94297632 .31(7 / 2) \quad \mathrm{pb}$ $52580.76329582 .69(5 / 2) \quad \mathrm{pb}$ $18763.68295757 .49(1 / 2)$ pb $\mathrm{N}$ $38152.74315026 .28(3 / 2) \quad \mathrm{pb}$ $75993.15352661 .04(3 / 2) \quad \mathrm{pb}$ $10194.49286471 .53(5 / 2) \quad \mathrm{pb}$ $56003.81331894 .85(3 / 2) \quad \mathrm{pb}$ $56003.81331765 .63(5 / 2)$ pb $\mathrm{N}$ $18763.68294367 .91(3 / 2) \quad \mathrm{pb}$ $78845.26 \quad 354205.09(5 / 2) \quad \mathrm{pb}$ $43289.57318483 .31(9 / 2) \quad \mathrm{pb}$ 


\begin{tabular}{|c|c|c|c|c|c|c|c|c|c|c|}
\hline 334 & 561 & 363.4123 & 275169.55 & 363.4110 & 0.0013 & -1.01 & 0.0013 & 6 & 9 & $5 d^{32} P_{1 / 2}$ \\
\hline 1398 & 1255 & 363.7507 & 274913.56 & 363.7517 & 0.0010 & 0.74 & -0.0010 & 7 & 11 & $5 d^{34} F_{9 / 2}$ \\
\hline 421 & 829 & 363.8232 & 274858.78 & 363.8252 & 0.0009 & 1.47 & -0.0020 & 9 & 16 & $5 d^{34} F_{5 / 2}$ \\
\hline 25 & 207 & 364.5446 & 274314.86 & 364.5443 & 0.0011 & -0.24 & 0.0003 & 5 & 9 & $5 d^{32} G_{9 / 2}$ \\
\hline 138 & 173 & 365.0146 & 273961.64 & 365.0189 & 0.0009 & 3.21 & -0.0043 & 8 & 16 & $5 d^{32} G_{7 / 2}$ \\
\hline 861 & 971 & 365.2103 & 273814.84 & 365.2090 & 0.0017 & -0.94 & 0.0013 & 6 & 7 & $5 d^{32} D 1_{5 / 2}$ \\
\hline 1266 & 1253 & 365.2345 & 273796.70 & 365.2351 & 0.0012 & 0.43 & -0.0006 & 7 & 12 & $5 d^{34} F_{3 / 2}$ \\
\hline 44 & 238 & 365.2907 & 273754.57 & 365.2921 & 0.0011 & 1.03 & -0.0014 & 7 & 9 & $5 d^{34} P_{1 / 2}$ \\
\hline 209 & 195 & 365.5242 & 273579.70 & 365.5253 & 0.0010 & 0.81 & -0.0011 & 10 & 15 & $5 d^{32} P_{3 / 2}$ \\
\hline 40 & 76 & 365.9350 & 273272.58 & 365.9349 & 0.0009 & -0.08 & 0.0001 & 10 & 16 & $5 d^{32} F_{5 / 2}$ \\
\hline 107 & 128 & 366.6245 & 272758.64 & 366.6256 & 0.0008 & 0.83 & -0.0011 & 10 & 18 & $5 d^{32} D 2_{5 / 2}$ \\
\hline 310 & 543 & 367.1592 & 272361.42 & 367.1558 & 0.0012 & -2.54 & 0.0034 & 9 & 9 & \\
\hline 157 & 288 & 367.2256 & 272312.17 & 367.2300 & 0.0010 & 3.27 & -0.0044 & 13 & 16 & $5 d^{32} G_{7 / 2}$ \\
\hline 103 & 125 & 367.2670 & 272281.47 & 367.2630 & 0.0009 & -2.99 & 0.0040 & 8 & 17 & $5 d^{34} P_{5 / 2}$ \\
\hline 146 & 364 & 367.3135 & 272247.00 & 367.3145 & 0.0010 & 0.77 & -0.0010 & 8 & 12 & $5 d^{32} H_{9 / 2}$ \\
\hline 431 & 269 & 368.5040 & 271367.47 & 368.5052 & 0.0011 & 0.89 & -0.0012 & 10 & 10 & $5 d^{32} D 1_{3 / 2}$ \\
\hline 98 & 140 & 369.5048 & 270632.48 & 369.5015 & 0.0009 & -2.45 & 0.0033 & 13 & 17 & $5 d^{34} P_{5 / 2}$ \\
\hline 34 & 32 & 370.7093 & 269753.15 & 370.7123 & 0.0009 & 2.20 & -0.0030 & 10 & 16 & $5 d^{32} F_{5 / 2}$ \\
\hline 218 & 300 & 371.2720 & 269344.31 & 371.2698 & 0.0009 & -1.57 & 0.0022 & 9 & 18 & $\mathrm{D} 2_{5 / 2}$ \\
\hline 556 & 326 & 371.4953 & 269182.41 & 371.4933 & 0.0012 & -1.42 & 0.0020 & 9 & 7 & $5 d^{32} D 1_{5 / 2}$ \\
\hline 546 & 742 & 371.9343 & 268864.69 & 371.9347 & 0.0013 & 0.32 & -0.0004 & 10 & 7 & $5 d^{32} H_{11 / 2}$ \\
\hline 234 & 327 & 372.1732 & 268692.10 & 372.1702 & 0.0009 & -2.14 & 0.0030 & 9 & 16 & $5 d^{3}{ }^{2} F_{5 / 2}$ \\
\hline 285 & 381 & 372.2199 & 268658.39 & 372.2177 & 0.0009 & -1.58 & 0.0022 & 6 & 18 & $5 d^{32} D 2_{5 / 2}$ \\
\hline 9 & 23 & 372.4320 & 268505.39 & 372.4296 & 0.0011 & -1.74 & 0.0024 & 5 & 16 & $5 d^{32} D 2_{3 / 2}$ \\
\hline 15 & 190 & 372.5099 & 268449.24 & 372.5085 & 0.0011 & -0.98 & 0.0014 & 4 & 14 & $5 d^{34} P_{3 / 2}$ \\
\hline 152 & 395 & 373.1769 & 267969.43 & 373.1789 & 0.0010 & 1.45 & -0.0020 & 9 & 15 & $5 d^{34} F_{7 / 2}$ \\
\hline 15 & 90 & 373.2855 & 267891.47 & 373.2831 & 0.0011 & -1.69 & 0.0024 & 10 & 15 & $5 d^{32} P_{3 / 2}$ \\
\hline 287 & 893 & 373.4949 & 267741.27 & 373.4947 & 0.0012 & -0.13 & 0.0002 & 6 & 12 & \\
\hline 493 & 819 & 373.5414 & 267707.94 & 373.5415 & 0.0010 & 0.10 & -0.0001 & 8 & 14 & $5 d^{3}{ }^{4} P_{3 / 2}$ \\
\hline 90 & 139 & 374.3179 & 267152.60 & 374.3164 & 0.0011 & -1.07 & 0.0015 & 10 & 9 & $5 d^{32} G_{9 / 2}$ \\
\hline 316 & 427 & 375.0319 & 266643.98 & 375.0330 & 0.0010 & 0.76 & -0.0011 & 11 & 17 & $5 d^{34} P_{5 / 2}$ \\
\hline 114 & 183 & 375.1740 & 266542.99 & 375.1735 & 0.0010 & -0.36 & 0.0005 & 10 & 14 & $5 d^{32} F_{7 / 2}$ \\
\hline 261 & 142 & 375.3793 & 266397.22 & 375.3775 & 0.0012 & -1.29 & 0.0018 & 6 & 7 & $5 d^{32} D 1_{5 / 2}$ \\
\hline 6 & 29 & 376.0539 & 265919.33 & 376.0579 & 0.0010 & 2.85 & -0.0040 & 10 & 16 & $5 d^{32} G_{7 / 2}$ \\
\hline 33 & 166 & 377.0590 & 265210.48 & 377.0598 & 0.0013 & 0.53 & -0.0008 & 4 & 9 & $5 d^{34} P_{1 / 2}$ \\
\hline 66 & 226 & 377.2329 & 265088.22 & 377.2334 & 0.0010 & 0.34 & -0.0005 & 9 & 16 & $5 d^{32} F_{5 / 2}$ \\
\hline 156 & 336 & 377.2715 & 265061.10 & 377.2732 & 0.0008 & 1.18 & -0.0017 & 12 & 18 & $5 d^{32} D 2_{5 / 2}$ \\
\hline 291 & 885 & 378.0852 & 264490.65 & 378.0892 & 0.0011 & 2.80 & -0.0040 & 9 & 11 & $5 d^{3}{ }^{4} F_{9 / 2}$ \\
\hline 107 & 366 & 378.1639 & 264435.61 & 378.1608 & 0.0010 & -2.18 & 0.0031 & 13 & 16 & $5 d^{32} G_{7 / 2}$ \\
\hline 67 & 245 & 378.2123 & 264401.77 & 378.2120 & 0.0010 & -0.21 & 0.0003 & 6 & 16 & $5 d^{32} E^{2}$ \\
\hline 673 & 956 & 378.4401 & 264242.61 & 378.4402 & 0.0010 & 0.10 & -0.0001 & 10 & 17 & $5 d^{34} P_{5 / 2}$ \\
\hline
\end{tabular}

$39855.71315026 .28(3 / 2) \quad \mathrm{pb}$ $20563.94295476 .76(9 / 2) \quad \mathrm{pb}$ $10194.49285051 .79(7 / 2) \quad \mathrm{pb}$ $51970.45326285 .54(11 / 2)$ pb 30, Pt VII $31715.83 \quad 305674.26(7 / 2) \quad \mathrm{pb}$ $78845.26 \quad 352661.04(3 / 2) \quad \mathrm{pb}$ $0.00 \quad 273796.26(3 / 2) \quad \mathrm{pb}$ $22003.95295757 .49(1 / 2) \quad \mathrm{pb}$ $56003.81329582 .69(5 / 2) \quad \mathrm{pb}$ $50624.30 \quad 323896.96(3 / 2) \quad \mathrm{pb}$ $46366.31 \quad 319124.12(7 / 2) \quad \mathrm{pb}$ $22003.95 \quad 294367.91(3 / 2) \quad \mathrm{pb}$ $31715.83304024 .73(5 / 2)$ pb N, 200 $33389.80305674 .26(7 / 2)$ pb $\mathrm{N}$ $33428.03 \quad 305674.26(7 / 2)$ pb 200, Pt VII $75993.15 \quad 347359.74(5 / 2) \quad \mathrm{pb}$ $33389.80 \quad 304024.73(5 / 2) \quad \mathrm{pb}$ $50624.30 \quad 320375.24(3 / 2) \quad \mathrm{pb}$ $46366.31315712 .19(5 / 2) \quad \mathrm{pb}$ $78845.26 \quad 348029.10(7 / 2) \quad \mathrm{pb}$ $43289.57312153 .94(9 / 2) \quad \mathrm{pb}$ $50624.30 \quad 319318.55(5 / 2) \quad \mathrm{pb}$ $46366.31315026 .28(3 / 2)$ pb Pt VII(100) $38152.74306659 .86(1 / 2)$ pb $\mathrm{N}$

$18763.68287213 .90(1 / 2)$ pb 100, Pt VII(100) $\begin{array}{lll}17083.81 & 285051.79(7 / 2) & \text { pb }\end{array}$

$56003.81323896 .96(3 / 2)$ pb 50, Pt VII $0.00267741 .40(5 / 2) \quad \mathrm{pb}$ $18763.68286471 .53(5 / 2) \quad \mathrm{pb}$ $51970.45 \quad 319124.12(7 / 2) \quad \mathrm{pb}$ $33389.80300033 .03(3 / 2) \quad p b$ $52580.76319124 .12(7 / 2) \quad \mathrm{pb}$ $78845.26 \quad 345243.77(7 / 2) \quad$ pb $31715.83 \quad 297632.31(7 / 2) \quad$ pb $* \mathrm{~N}$ $22003.95287213 .90(1 / 2) \quad \mathrm{pb}$ $50624.30315712 .19(5 / 2)$ pb Pt VII(50) $46366.31 \quad 311426.23(7 / 2) \quad \mathrm{pb}$ $20563.94285051 .79(7 / 2) \quad \mathrm{pb}$ $31715.83296153 .62(5 / 2) \quad \mathrm{pb}$ $50624.30 \quad 315026.28(3 / 2) \quad$ pb $33389.80 \quad 297632.31(7 / 2) \quad \mathrm{pb}$ 


\begin{tabular}{|c|c|c|c|c|c|c|c|c|c|c|}
\hline 3 & 71 & 379.3569 & 263604.01 & 379.3601 & 0.0010 & 2.23 & -0.0032 & 7 & 16 & $5 d^{34} F_{5 / 2}$ \\
\hline 56 & 33 & 379.6886 & 263373.72 & 379.6865 & 0.0012 & -1.46 & 0.0021 & 6 & 10 & $5 d^{32} \mathrm{D} 1_{3 / 2}$ \\
\hline 371 & 663 & 380.0378 & 263131.72 & 380.0382 & 0.0011 & 0.29 & -0.0004 & 9 & 14 & $5 d^{32}$ \\
\hline 68 & 242 & 380.5668 & 262765.96 & 380.5699 & 0.0009 & 2.14 & -0.0031 & 13 & 17 & \\
\hline 128 & 158 & 382.8810 & 261177.76 & 382.8822 & 0.0012 & 0.83 & -0.0012 & 9 & 10 & $5 d^{32} D 1_{3 / 2}$ \\
\hline 15 & 82 & 383.1754 & 260977.09 & 383.1739 & 0.0010 & -1.02 & 0.0015 & 9 & 17 & \\
\hline 502 & 835 & 383.4309 & 260803.18 & 383.4327 & 0.0009 & 1.26 & -0.0018 & 12 & 16 & \\
\hline 106 & 410 & 384.2636 & 260238.02 & 384.2661 & 0.0012 & 1.67 & -0.0025 & 4 & 10 & 1 \\
\hline 5 & 30 & 384.3581 & 260174.04 & 384.3533 & 0.0014 & -3.27 & 0.0048 & 11 & 9 & \\
\hline 63 & 250 & 385.0425 & 259711.59 & 385.0472 & & 3.20 & -0.0047 & 9 & 15 & \\
\hline 106 & 288 & 85.2502 & 59571.57 & 385.2478 & 0.0010 & -1.60 & 0.0024 & 10 & 14 & \\
\hline 56 & 172 & 385.4197 & 259457.42 & 385.4221 & 0.0012 & 1.64 & -0.0024 & 12 & 9 & \\
\hline 114 & 359 & 386.20 & 258926.96 & 386.2244 & 0.0012 & 10.11 & -0.0151 & 12 & 10 & $1_{3 / 2}$ \\
\hline 60 & 182 & 386.3293 & 2588 & 386.3309 & 0.0010 & 1.06 & -0.0016 & 12 & 14 & \\
\hline 139 & 244 & 777 & 2583 & 095 & & 1.22 & -0.0018 & 9 & 7 & $\mathrm{D} 1_{5 / 2}$ \\
\hline 23 & 138 & 87.5954 & 58001.00 & 387.5956 & 0.0009 & 0.11 & -0.0002 & 13 & 16 & $2_{3 / 2}$ \\
\hline 22 & 131 & 390.7 & 2559 & 390. & & 1.60 & -0.0024 & 7 & 9 & \\
\hline 64 & 331 & 392. & 2550 & 392 & & 2.51 & -0. & 7 & 14 & \\
\hline 40 & 530 & 392.9018 & 2545 & 392.9085 & 0.0015 & 4.33 & -0.0067 & 9 & 9 & $5 d^{32}$ \\
\hline 15 & 84 & 394. & 2536 & 2181 & & -0.03 & & 11 & 18 & $\mathrm{D} 2_{5 / 2}$ \\
\hline 55 & 195 & 394. & 2534 & 323 & & 0.14 & -0.0002 & 13 & 16 & \\
\hline 28 & 230 & & 533 & & & 0.42 & & 9 & 16 & \\
\hline 52 & 664 & 395.1143 & 253091.32 & 395.1293 & & 9.59 & -0.0 & 8 & 17 & \\
\hline 105 & 497 & 396. & 2521 & 309 & & 1.69 & -0.00 & 7 & 7 & $\mathrm{H}_{11 / 2}$ \\
\hline 19 & 370 & 397. & 2517 & 1527 & & -0.06 & & 7 & 9 & ${ }^{4} \mathrm{P}_{1 / 2}$ \\
\hline 40 & 440 & 397.4204 & 2516 & 397.4188 & & -1.04 & 0.0016 & 9 & 12 & $5 d^{32} H_{9 / 2}$ \\
\hline 16 & 115 & 397.7 & 25144 & 397.7029 & & 0.14 & -0.0002 & 13 & 14 & \\
\hline 10 & 89 & 398.9448 & 1.24 & 398.9506 & & 3.65 & & 6 & 15 & \\
\hline 13 & 63 & & 2497 & & & 1.18 & & 13 & 18 & \\
\hline 83 & 934 & 400.9309 & 249419.54 & 400.9483 & & 10.82 & & 11 & 16 & $5 d^{32} F_{5 / 2}$ \\
\hline 19 & 357 & & 2489 & 401 & & 2.52 & -0. & 6 & 14 & $5 d^{34} P_{3 / 2}$ \\
\hline 5 & 132 & & 2480 & 1918 & & 3.93 & & 13 & 15 & $\mathrm{P}_{3 / 2}$ \\
\hline 22 & 98 & & 247999.88 & 403.2232 & & -1.72 & & 9 & 18 & $5 d^{32} D 2_{5 / 2}$ \\
\hline 19 & 49 & & 247904.46 & & & 0.65 & -0.0011 & 10 & 10 & \\
\hline 44 & 219 & 404.8479 & 247006.35 & 404.8452 & & -1.66 & & 10 & 16 & \\
\hline 32 & 815 & & 24505 & 408.0774 & & 1.08 & -0.00 & 10 & 14 & \\
\hline 29 & 241 & 410.6665 & 243506.59 & 410.6670 & & 0.29 & & 7 & 9 & $5 d^{32} G_{9 / 2}$ \\
\hline 14 & 72 & 416.4833 & 240105.67 & 416.4841 & & 0.45 & -0.0008 & 8 & 18 & $5 d^{32} D 2_{5 / 2}$ \\
\hline 11 & 149 & 417.0903 & 239756.23 & 417.0947 & & 2.55 & -0.0044 & 7 & 15 & \\
\hline 11 & 166 & & 235847.89 & 424.0033 & & 0.66 & -0.0012 & 8 & 16 & \\
\hline 9 & 117 & 424.3741 & 235641.15 & 424.3698 & 0.0012 & -2.38 & 0.0043 & 7 & 16 & \\
\hline
\end{tabular}

$10194.49273796 .26(3 / 2) \quad$ pb $\mathrm{N}$ $75993.15339368 .34(1 / 2)$ pb $\mathrm{N}$ $52580.76 \quad 315712.19(5 / 2) \quad \mathrm{pb}$ $33389.80296153 .62(5 / 2)$ pb $\mathrm{N}$ $75993.15337170 .08(5 / 2)$ pb $\mathrm{N}$ $33389.80 \quad 294367.91(3 / 2) \quad$ pb $\quad \mathrm{N}$

$50624.30 \quad 311426.23(7 / 2)$ pb 550, Pt VII(300)

$75993.15336229 .51(1 / 2)$ pb N, 250

$39855.71300033 .03(3 / 2) \quad \mathrm{pb} * \mathrm{~N}$

$56003.81315712 .19(5 / 2) \quad \mathrm{pb} * \mathrm{bl}$

$52580.76312153 .94(9 / 2) \quad \mathrm{pb}$

$51970.45311426 .23(7 / 2) \quad \mathrm{pb}$

$75993.15334910 .00(3 / 2) \quad$ pb $* \mathrm{~N}, 150, \mathrm{bl}(\mathrm{C}$ III)

$52580.76 \quad 311426.23(7 / 2) \quad \mathrm{pb}$

$78845.26337170 .08(5 / 2)$ pb $\mathrm{N}$

$38152.74296153 .62(5 / 2)$ pb $\mathrm{N}$

$39855.71295757 .49(1 / 2) \quad \mathrm{pb}$

$18763.68273796 .26(3 / 2) \quad \mathrm{pb}$

$39855.71294367 .91(3 / 2) \quad \mathrm{pb} * \mathrm{~N}, 150, \mathrm{~m}(\mathrm{Pt}$ VII $)$

$46366.31300033 .03(3 / 2)$ pb $\mathrm{N}$

$50624.30304024 .73(5 / 2)$ pb N

$31715.83285051 .79(7 / 2) \quad$ pb $\mathrm{N}$

$33389.80286471 .53(5 / 2) \quad$ pb $*$ N, 200, m(Pt VII) $43289.57295476 .76(9 / 2) \quad \mathrm{pb}$

$22003.95273796 .26(3 / 2)$ pb 150, Pt VII $(250)$

$33428.03285051 .79(7 / 2)$ pb N, 300

$52580.76304024 .73(5 / 2)$ pb $\mathrm{N}$

$17083.81267741 .40(5 / 2) \quad \mathrm{pb} N$

$46366.31296153 .62(5 / 2) \quad \mathrm{pb} \mathrm{N}$

$50624.30300033 .03(3 / 2) \quad$ pb $* \mathrm{~N}, 200, \mathrm{~m}(\mathrm{Pt} \mathrm{VII})$

$18763.68267741 .40(5 / 2) \quad$ pb N

$56003.81304024 .73(5 / 2) \quad \mathrm{pb} * \mathrm{~N}$

$46366.31294367 .91(3 / 2)$ pb $\mathrm{N}$

$75993.15323896 .96(3 / 2) \quad$ pb $\mathrm{N}$

$50624.30 \quad 297632.31(7 / 2) \quad$ pb N

$52580.76297632 .31(7 / 2) \quad$ pb $* \mathrm{~N}, 150, \mathrm{~m}(\mathrm{Pt}$ VII $)$

$51970.45295476 .76(9 / 2) \quad$ pb $\mathrm{N}$

$46366.31286471 .53(5 / 2)$ pb $\mathrm{N}$

$56003.81295757 .49(1 / 2)$ pb $\mathrm{N}$

$50624.30286471 .53(5 / 2)$ pb $\mathrm{N}$

$38152.74273796 .26(3 / 2)$ pb $\mathrm{N}$ 


\begin{tabular}{|c|c|c|c|c|c|c|c|c|c|c|c|c|c|c|}
\hline 12 & 113 & 430.1607 & 232471.26 & 430.1611 & 0.0012 & 0.23 & -0.0004 & 9 & 14 & $5 d^{32} F_{7 / 2}$ & 52580.76 & $285051.79(7 / 2)$ & $\mathrm{pb}$ & $\mathrm{N}$ \\
\hline 9 & 265 & 435.5615 & 229588.70 & 435.5616 & 0.0012 & 0.04 & -0.0001 & 6 & 16 & $5 d^{32} D 2_{3 / 2}$ & 38152.74 & $267741.40(5 / 2)$ & $\mathrm{pb}$ & $\mathrm{N}, 150$ \\
\hline 17 & 110 & 452.1030 & 221188.53 & 452.1046 & 0.0017 & 0.77 & -0.0016 & 11 & 7 & $5 d^{32} \mathrm{D} 1_{5 / 2}$ & 78845.26 & $300033.03(3 / 2)$ & $\mathrm{pb}$ & $\mathrm{N}$ \\
\hline 11 & 95 & 692.8336 & 144334.80 & 692.8289 & 0.0016 & -0.97 & 0.0047 & 10 & 7 & $5 d^{2} 6 s\left({ }^{3} F\right)^{4} F_{3 / 2}$ & 176039.47 & $320375.24(3 / 2)$ & $\mathrm{pc}$ & \\
\hline 40 & 297 & 710.6802 & 140710.27 & 710.6794 & 0.0016 & -0.15 & 0.0008 & 10 & 10 & $5 d^{2} 6 s\left({ }^{3} F\right)^{4} F_{5 / 2}$ & 179664.83 & $320375.24(3 / 2)$ & pc & \\
\hline 19 & 72 & 716.0595 & 139653.20 & 716.0568 & 0.0020 & -0.52 & 0.0027 & 9 & 10 & $5 d^{2} 6 s\left({ }^{3} F\right)^{4} F_{5 / 2}$ & 179664.83 & $319318.55(5 / 2)$ & $\mathrm{pc} *$ & \\
\hline 62 & 263 & 719.9588 & 138896.84 & 719.9600 & 0.0018 & 0.23 & -0.0012 & 10 & 10 & $5 d^{2} 6 s\left({ }^{3} F\right)^{2} F_{7 / 2}$ & 208463.13 & $347359.74(5 / 2)$ & $\mathrm{pc}$ & \\
\hline 19 & 126 & 723.2681 & 138261.32 & 723.2674 & 0.0016 & -0.13 & 0.0007 & 12 & 12 & $5 d^{2} 6 s\left({ }^{3} F\right)^{2} F_{5 / 2}$ & 196648.56 & $334910.00(3 / 2)$ & $\mathrm{pc}$ & \\
\hline 30 & 146 & 725.2062 & 137891.82 & 725.2026 & 0.0015 & -0.69 & 0.0036 & 10 & 11 & $5 d^{2} 6 s\left({ }^{3} F\right)^{4} F_{7 / 2}$ & 191690.18 & $329582.69(5 / 2)$ & pc & \\
\hline 20 & 83 & 734.0878 & 136223.49 & 734.0865 & 0.0022 & -0.23 & 0.0013 & 10 & 4 & $5 \mathrm{~d}^{2} 6 \mathrm{~s}\left({ }^{3} \mathrm{P}\right){ }^{4} \mathrm{P}_{1 / 2}$ & 195671.13 & $331894.85(3 / 2)$ & $\mathrm{pc}$ & \\
\hline 26 & 210 & 738.0048 & 135500.47 & 738.0107 & 0.0019 & 1.09 & -0.0059 & 11 & 12 & $5 d^{2} 6 s\left({ }^{3} F\right)^{2} F_{5 / 2}$ & 196648.56 & $332147.94(7 / 2)$ & $\mathrm{pc} *$ & ${ }^{*} 100, \mathrm{Pt} \mathrm{V}$ \\
\hline 6 & 138 & 739.3880 & 135246.99 & 739.3918 & 0.0020 & 0.69 & -0.0038 & 10 & 12 & $5 d^{2} 6 s\left({ }^{3} F\right)^{2} F_{5 / 2}$ & 196648.56 & $331894.85(3 / 2)$ & $\mathrm{pc} *$ & \\
\hline 31 & 159 & 740.1000 & 135116.88 & 740.0989 & 0.0021 & -0.19 & 0.0011 & 10 & 12 & $5 d^{2} 6 s\left({ }^{3} F\right)^{2} F_{5 / 2}$ & 196648.56 & $331765.63(5 / 2)$ & $\mathrm{pc}$ & \\
\hline 44 & 143 & 753.3281 & 132744.29 & 753.3272 & 0.0023 & -0.16 & 0.0009 & 8 & 6 & $5 d^{2} 6 s\left({ }^{1} D\right)^{2} D_{5 / 2}$ & 221460.64 & $354205.09(5 / 2)$ & $\mathrm{pc}$ & \\
\hline 49 & 326 & 755.2342 & 132409.26 & 755.2374 & 0.0016 & 0.55 & -0.0032 & 7 & 11 & $5 d^{2} 6 s\left({ }^{3} F\right)^{4} F_{7 / 2}$ & 191690.18 & $324098.89(7 / 2)$ & $\mathrm{pc}$ & \\
\hline 230 & 633 & 756.1959 & 132240.86 & 756.1981 & 0.0022 & 0.38 & -0.0022 & 8 & 8 & $5 d^{2} 6 s\left({ }^{1} G\right)^{2} G_{7 / 2}$ & 221964.61 & $354205.09(5 / 2)$ & $\mathrm{pc}$ & \\
\hline 15 & 107 & 756.4843 & 132190.45 & 756.4816 & 0.0017 & -0.46 & 0.0027 & 12 & 9 & $5 d^{2} 6 s\left({ }^{3} \mathrm{P}\right){ }^{4} \mathrm{P}_{3 / 2}$ & 202719.09 & $334910.00(3 / 2)$ & $\mathrm{pc}$ & $2 d$ ord \\
\hline 10 & 58 & 758.9515 & 131760.73 & 758.9476 & 0.0020 & -0.68 & 0.0039 & 12 & 10 & $5 d^{2} 6 s\left({ }^{3} F\right)^{4} F_{5 / 2}$ & 179664.83 & $311426.23(7 / 2)$ & pc * & \\
\hline 24 & 254 & 759.7703 & 131618.73 & 759.7714 & 0.0017 & 0.18 & -0.0011 & 12 & 9 & $5 d^{2} 6 s\left({ }^{3} P\right)^{4} P_{5 / 2}$ & 203291.46 & $334910.00(3 / 2)$ & $\mathrm{pc}$ & \\
\hline 97 & 583 & 761.0882 & 131390.82 & 761.0850 & 0.0018 & -0.55 & 0.0032 & 11 & 7 & $5 d^{2} 6 s\left({ }^{3} F\right)^{4} F_{9 / 2}$ & 200756.58 & $332147.94(7 / 2)$ & $\mathrm{pc}$ & \\
\hline 24 & 168 & 761.6884 & 131287.28 & 761.6868 & 0.0022 & -0.28 & 0.0016 & 6 & 10 & $5 d^{2} 6 s\left({ }^{1} D\right)^{2} D_{3 / 2}$ & 208080.78 & $339368.34(1 / 2)$ & pc & $2 \mathrm{~d}$ ord \\
\hline 106 & 573 & 765.5763 & 130620.55 & 765.5773 & 0.0030 & 0.16 & -0.0009 & 5 & 7 & $5 d^{2} 6 s\left({ }^{3} F\right)^{4} F_{3 / 2}$ & 176039.47 & $306659.86(1 / 2)$ & $\mathrm{pc}$ & \\
\hline 218 & 703 & 771.4068 & 129633.29 & 771.4069 & 0.0021 & 0.02 & -0.0001 & 8 & 7 & $5 d^{2} 6 s\left({ }^{3} F\right)^{4} F_{9 / 2}$ & 200756.58 & $330389.85(9 / 2)$ & $\mathrm{pc}$ & \\
\hline 54 & 725 & 774.1501 & 129173.92 & 774.1390 & 0.0022 & -1.85 & 0.0111 & 10 & 9 & $5 d^{2} 6 s\left({ }^{3} P\right)^{4} P_{3 / 2}$ & 202719.09 & $331894.85(3 / 2)$ & $\mathrm{pc} *$ & $200, \mathrm{~m}$ \\
\hline 133 & 725 & 774.1501 & 129173.92 & 774.1507 & 0.0021 & 0.11 & -0.0006 & 9 & 6 & $5 d^{2} 6 s\left({ }^{1} G\right)^{2} G_{9 / 2}$ & 218855.29 & $348029.10(7 / 2)$ & $\mathrm{pc}$ & \\
\hline 94 & 354 & 774.6579 & 129089.24 & 774.6575 & 0.0022 & -0.06 & 0.0004 & 9 & 10 & $5 d^{2} 6 s\left({ }^{1} D\right)^{2} D_{3 / 2}$ & 208080.78 & $337170.08(5 / 2)$ & $\mathrm{pc}$ & \\
\hline 250 & 973 & 774.9218 & 129045.28 & 774.9142 & 0.0030 & -1.26 & 0.0076 & 10 & 9 & $5 d^{2} 6 s\left({ }^{3} P\right)^{4} P_{3 / 2}$ & 202719.09 & $331765.63(5 / 2)$ & $\mathrm{pc} *$ & ${ }^{*} \mathrm{Pt}$ IV \\
\hline 176 & 633 & 776.0593 & 128856.13 & 776.0572 & 0.0019 & -0.35 & 0.0021 & 11 & 9 & $5 d^{2} 6 s\left({ }^{3} P\right)^{4} P_{5 / 2}$ & 203291.46 & $332147.94(7 / 2)$ & $\mathrm{pc}$ & \\
\hline 155 & 585 & 776.9583 & 128707.04 & 776.9588 & 0.0022 & 0.08 & -0.0005 & 9 & 10 & $5 d^{2} 6 s\left({ }^{3} F\right)^{2} F_{7 / 2}$ & 208463.13 & $337170.08(5 / 2)$ & $\mathrm{pc}$ & \\
\hline 48 & 238 & 778.3652 & 128474.40 & 778.3666 & 0.0022 & 0.23 & -0.0014 & 10 & 9 & $5 d^{2} 6 s\left({ }^{3} \mathrm{P}\right)^{4} \mathrm{P}_{5 / 2}$ & 203291.46 & $331765.63(5 / 2)$ & $\mathrm{pc}$ & \\
\hline 122 & 686 & 779.8748 & 128225.71 & 779.8741 & 0.0023 & -0.12 & 0.0007 & 10 & 4 & $5 d^{2} 6 s\left({ }^{3} P\right){ }^{4} P_{1 / 2}$ & 195671.13 & $323896.96(3 / 2)$ & pc & \\
\hline 20 & 238 & 780.3425 & 128148.86 & 780.3433 & 0.0022 & 0.12 & -0.0008 & 4 & 10 & $5 d^{2} 6 s\left({ }^{1} D\right)^{2} D_{3 / 2}$ & 208080.78 & $336229.51(1 / 2)$ & $\mathrm{pc}$ & $2 \mathrm{~d}$ ord \\
\hline 130 & 639 & 781.3402 & 127985.22 & 781.3400 & 0.0018 & -0.04 & 0.0002 & 13 & 7 & $5 d^{2} 6 s\left({ }^{3} F\right)^{4} F_{3 / 2}$ & 176039.47 & $304024.73(5 / 2)$ & $\mathrm{pc}$ & Pt V(100) \\
\hline 61 & 370 & 783.5289 & 127627.71 & 783.5249 & 0.0021 & -0.65 & 0.0040 & 9 & 11 & $5 d^{2} 6 s\left({ }^{3} F\right)^{4} F_{7 / 2}$ & 191690.18 & $319318.55(5 / 2)$ & $\mathrm{pc}$ & \\
\hline 148 & 685 & 784.7210 & 127433.83 & 784.7203 & 0.0020 & -0.11 & 0.0007 & 10 & 11 & $5 d^{2} 6 s\left({ }^{3} F\right)^{4} F_{7 / 2}$ & 191690.18 & $319124.12(7 / 2)$ & $\mathrm{pc}$ & \\
\hline 38 & 171 & 785.8649 & 127248.34 & 785.8645 & 0.0022 & -0.07 & 0.0004 & 10 & 12 & $5 d^{2} 6 s\left({ }^{3} F\right)^{2} F_{5 / 2}$ & 196648.56 & $323896.96(3 / 2)$ & $\mathrm{pc}$ & \\
\hline 53 & 382 & 788.4618 & 126829.23 & 788.4618 & 0.0017 & 0.00 & 0.0000 & 12 & 10 & $5 d^{2} 6 s\left({ }^{1} D\right)^{2} D_{3 / 2}$ & 208080.78 & $334910.00(3 / 2)$ & $\mathrm{pc}$ & \\
\hline 382 & 1094 & 788.6852 & 126793.30 & 788.6863 & 0.0018 & 0.17 & -0.0011 & 7 & 11 & $5 d^{2} 6 s\left({ }^{3} F\right)^{4} F_{7 / 2}$ & 191690.18 & $318483.31(9 / 2)$ & $\mathrm{pc}$ & \\
\hline 153 & 855 & 789.9839 & 126584.86 & 789.9803 & 0.0022 & -0.58 & 0.0037 & 5 & 7 & $5 d^{2} 6 s\left({ }^{3} F\right)^{4} F_{3 / 2}$ & 176039.47 & $302624.91(3 / 2)$ & $\mathrm{pc}$ & \\
\hline 173 & 1150 & 790.0962 & 126566.87 & 790.0863 & 0.0030 & -1.59 & 0.0099 & 9 & 6 & $5 d^{2} 6 s\left({ }^{1} D\right)^{2} D_{5 / 2}$ & 221460.64 & $348029.10(7 / 2)$ & $\mathrm{pc} *$ & $800, \mathrm{~m}(\mathrm{O}$ IV $)$ \\
\hline 668 & 1174 & 791.6487 & 126318.66 & 791.6496 & 0.0030 & 0.14 & -0.0009 & 3 & 6 & $5 d^{2} 6 s\left({ }^{1} G\right)^{2} G_{9 / 2}$ & 218855.29 & $345173.80(11 / 2)$ & $\mathrm{pc}$ & \\
\hline
\end{tabular}




\begin{tabular}{|c|c|c|c|c|c|c|c|c|c|c|c|c|c|c|}
\hline 81 & 293 & 791.8234 & 126290.79 & 791.8206 & 0.0018 & -0.45 & 0.0028 & 10 & 9 & $5 d^{2} 6 s\left({ }^{3} P\right)^{4} P_{5 / 2}$ & 203291.46 & $329582.69(5 / 2)$ & $\mathrm{pc}$ & \\
\hline 48 & 420 & 791.9939 & 126263.60 & 791.9968 & 0.0023 & 0.46 & -0.0029 & 6 & 4 & $5 d^{2} 6 s\left({ }^{3} \mathrm{P}\right)^{2} \mathrm{P}_{1 / 2}$ & 213105.19 & $339368.34(1 / 2)$ & $\mathrm{pc}$ & \\
\hline 116 & 404 & 793.2447 & 126064.50 & 793.2448 & 0.0022 & 0.02 & -0.0001 & 9 & 8 & $5 d^{2} 6 s\left({ }^{1} G\right)^{2} G_{7 / 2}$ & 221964.61 & $348029.10(7 / 2)$ & $\mathrm{pc}$ & \\
\hline 339 & 1127 & 793.5883 & 126009.92 & 793.5914 & 0.0018 & 0.49 & -0.0031 & 8 & 10 & $5 d^{2} 6 s\left({ }^{3} F\right)^{4} F_{5 / 2}$ & 179664.83 & $305674.26(7 / 2)$ & $\mathrm{pc}$ & \\
\hline 113 & 367 & 794.2872 & 125899.04 & 794.2869 & 0.0022 & -0.05 & 0.0003 & 10 & 6 & $5 d^{2} 6 s\left({ }^{1} D\right)^{2} D_{5 / 2}$ & 221460.64 & $347359.74(5 / 2)$ & pc & \\
\hline 652 & 1160 & 796.6281 & 125529.09 & 796.6289 & 0.0030 & 0.13 & -0.0008 & 5 & 7 & $5 d^{2} 6 s\left({ }^{3} F\right){ }^{4} F_{9 / 2}$ & 200756.58 & $326285.54(11 / 2)$ & pc & \\
\hline 71 & 395 & 797.4799 & 125395.01 & 797.4792 & 0.0020 & -0.12 & 0.0007 & 10 & 8 & $5 d^{2} 6 s\left({ }^{1} G\right)^{2} G_{7 / 2}$ & 221964.61 & $347359.74(5 / 2)$ & $\mathrm{pc}$ & Pt IV(150) \\
\hline 47 & 305 & 801.8960 & 124704.45 & 801.8982 & 0.0023 & 0.34 & -0.0022 & 10 & 4 & $5 d^{2} 6 s\left({ }^{3} P\right){ }^{4} P_{1 / 2}$ & 195671.13 & $320375.24(3 / 2)$ & $\mathrm{pc}$ & \\
\hline 137 & 719 & 804.1162 & 124360.14 & 804.1177 & 0.0019 & 0.23 & -0.0015 & 13 & 10 & $5 d^{2} 6 s\left({ }^{3} F\right)^{4} F_{5 / 2}$ & 179664.83 & $304024.73(5 / 2)$ & $\mathrm{pc}$ & \\
\hline 83 & 264 & 807.6574 & 123814.88 & 807.6626 & 0.0021 & 0.80 & -0.0052 & 10 & 10 & $5 d^{2} 6 s\left({ }^{1} D\right)^{2} D_{3 / 2}$ & 208080.78 & $331894.85(3 / 2)$ & $\mathrm{pc}$ & \\
\hline 241 & 673 & 807.8641 & 123783.20 & 807.8646 & 0.0024 & 0.07 & -0.0005 & 6 & 6 & $5 d^{2} 6 s\left({ }^{1} D\right)^{2} D_{5 / 2}$ & 221460.64 & $345243.77(7 / 2)$ & $\mathrm{pc}$ & \\
\hline 97 & 553 & 808.2319 & 123726.87 & 808.2331 & 0.0020 & 0.18 & -0.0012 & 10 & 12 & $5 d^{2} 6 s\left({ }^{3} F\right)^{2} F_{5 / 2}$ & 196648.56 & $320375.24(3 / 2)$ & $\mathrm{pc}$ & \\
\hline 115 & 648 & 808.5009 & 123685.70 & 808.5067 & 0.0019 & 0.88 & -0.0058 & 11 & 10 & $5 d^{2} 6 s\left({ }^{3} F\right)^{2} F_{7 / 2}$ & 208463.13 & $332147.94(7 / 2)$ & $\mathrm{pc}$ & \\
\hline 43 & 320 & 810.7542 & 123341.95 & 810.7518 & 0.0018 & -0.36 & 0.0024 & 7 & 7 & $5 d^{2} 6 s\left({ }^{3} F\right)^{4} F_{9 / 2}$ & 200756.58 & $324098.89(7 / 2)$ & $\mathrm{pc}$ & \\
\hline 129 & 445 & 811.1661 & 123279.31 & 811.1671 & 0.0023 & 0.16 & -0.0010 & 6 & 8 & $5 d^{2} 6 s\left({ }^{1} G\right)^{2} G_{7 / 2}$ & 221964.61 & $345243.77(7 / 2)$ & $\mathrm{pc}$ & \\
\hline 57 & 377 & 811.9161 & 123165.43 & 811.9165 & 0.0022 & 0.06 & -0.0004 & 4 & 9 & $5 d^{2} 6 s\left({ }^{3} P\right)^{4} P_{3 / 2}$ & 202719.09 & $325884.46(1 / 2)$ & $\mathrm{pc}$ & \\
\hline 11 & 71 & 812.1880 & 123124.20 & 812.1872 & 0.0024 & -0.12 & 0.0008 & 4 & 4 & $5 d^{2} 6 s\left({ }^{3} P\right)^{2} P_{1 / 2}$ & 213105.19 & $336229.51(1 / 2)$ & $\mathrm{pc}$ & \\
\hline 29 & 161 & 813.2674 & 122960.79 & 813.2720 & 0.0023 & 0.70 & -0.0046 & 5 & 10 & $5 d^{2} 6 s\left({ }^{3} F\right)^{4} F_{5 / 2}$ & 179664.83 & $302624.91(3 / 2)$ & pc & \\
\hline 150 & 572 & 815.1914 & 122670.58 & 815.1953 & 0.0022 & 0.59 & -0.0039 & 9 & 12 & $5 d^{2} 6 s\left({ }^{3} F\right)^{2} F_{5 / 2}$ & 196648.56 & $319318.55(5 / 2)$ & $\mathrm{pc}$ & \\
\hline 200 & 792 & 816.4873 & 122475.88 & 816.4894 & 0.0022 & 0.32 & -0.0021 & 10 & 12 & $5 d^{2} 6 s\left({ }^{3} F\right)^{2} F_{5 / 2}$ & 196648.56 & $319124.12(7 / 2)$ & $\mathrm{pc}$ & \\
\hline 280 & 728 & 820.1639 & 121926.85 & 820.1647 & 0.0023 & 0.12 & -0.0008 & 8 & 10 & $5 d^{2} 6 s\left({ }^{3} F\right)^{2} F_{7 / 2}$ & 208463.13 & $330389.85(9 / 2)$ & $\mathrm{pc}$ & \\
\hline 92 & 435 & 820.9841 & 121805.04 & 820.9856 & 0.0022 & 0.23 & -0.0016 & 12 & 4 & $5 d^{2} 6 s\left({ }^{3} \mathrm{P}\right)^{2} \mathrm{P}_{1 / 2}$ & 213105.19 & $334910.00(3 / 2)$ & $\mathrm{pc}$ & \\
\hline 75 & 347 & 823.0308 & 121502.14 & 823.0323 & 0.0020 & 0.22 & -0.0015 & 10 & 10 & $5 d^{2} 6 s\left({ }^{1} D\right)^{2} D_{3 / 2}$ & 208080.78 & $329582.69(5 / 2)$ & $\mathrm{pc}$ & \\
\hline 203 & 647 & 823.7444 & 121396.88 & 823.7484 & 0.0022 & 0.59 & -0.0040 & 7 & 6 & $5 d^{2} 6 s\left({ }^{1} G\right)^{2} G_{9 / 2}$ & 218855.29 & $340251.58(9 / 2)$ & $\mathrm{pc}$ & \\
\hline 74 & 243 & 825.6275 & 121120.00 & 825.6305 & 0.0019 & 0.44 & -0.0030 & 10 & 10 & $5 d^{2} 6 s\left({ }^{3} F\right)^{2} F_{7 / 2}$ & 208463.13 & $329582.69(5 / 2)$ & pc & \\
\hline 215 & 657 & 827.7596 & 120808.02 & 827.7637 & 0.0019 & 0.59 & -0.0041 & 7 & 9 & $5 d^{2} 6 s\left({ }^{3} P\right){ }^{4} P_{5 / 2}$ & 203291.46 & $324098.89(7 / 2)$ & $\mathrm{pc}$ & \\
\hline 114 & 766 & 830.1256 & 120463.70 & 830.1252 & 0.0018 & -0.06 & 0.0004 & 10 & 11 & $5 d^{2} 6 s\left({ }^{3} F\right)^{4} F_{7 / 2}$ & 191690.18 & $312153.94(9 / 2)$ & $\mathrm{pc}$ & $2 d$ ord \\
\hline 19 & 131 & 832.5391 & 120114.48 & 832.5414 & 0.0019 & 0.33 & -0.0023 & 13 & 7 & $5 d^{2} 6 s\left({ }^{3} F\right)^{4} F_{3 / 2}$ & 176039.47 & $296153.62(5 / 2)$ & $\mathrm{pc}$ & \\
\hline 31 & 111 & 841.8274 & 118789.20 & 841.8241 & 0.0023 & -0.46 & 0.0033 & 10 & 4 & $5 d^{2} 6 s\left({ }^{3} \mathrm{P}\right)^{2} \mathrm{P}_{1 / 2}$ & 213105.19 & $331894.85(3 / 2)$ & $\mathrm{pc}$ & \\
\hline 237 & 840 & 845.4056 & 118286.42 & 845.4016 & 0.0023 & -0.55 & 0.0040 & 7 & 8 & $5 d^{2} 6 s\left({ }^{1} G\right)^{2} G_{7 / 2}$ & 221964.61 & $340251.58(9 / 2)$ & pc & \\
\hline 12 & 68 & 847.6912 & 117967.49 & 847.6912 & 0.0020 & 0.00 & 0.0000 & 10 & 10 & $5 d^{2} 6 s\left({ }^{3} F\right)^{4} F_{5 / 2}$ & 179664.83 & $297632.31(7 / 2)$ & $\mathrm{pc}$ & \\
\hline 19 & 74 & 848.8701 & 117803.65 & 848.8699 & 0.0023 & -0.03 & 0.0002 & 4 & 10 & $5 \mathrm{~d}^{2} 6 \mathrm{~s}\left({ }^{1} \mathrm{D}\right)^{2} \mathrm{D}_{3 / 2}$ & 208080.78 & $325884.46(1 / 2)$ & $\mathrm{pc}$ & \\
\hline 44 & 292 & 849.4234 & 117726.92 & 849.4248 & 0.0021 & 0.19 & -0.0013 & 7 & 7 & $5 d^{2} 6 s\left({ }^{3} F\right)^{4} F_{9 / 2}$ & 200756.58 & $318483.31(9 / 2)$ & $\mathrm{pc}$ & \\
\hline 21 & 48 & 861.8734 & 116026.32 & 861.8677 & 0.0030 & -0.77 & 0.0057 & 9 & 9 & $5 d^{2} 6 s\left({ }^{3} P\right){ }^{4} P_{5 / 2}$ & 203291.46 & $319318.55(5 / 2)$ & $\mathrm{pc} *$ & \\
\hline 73 & 171 & 864.7889 & 115635.16 & 864.7844 & 0.0019 & -0.60 & 0.0045 & 7 & 10 & $5 d^{2} 6 s\left({ }^{3} F\right)^{2} F_{7 / 2}$ & 208463.13 & $324098.89(7 / 2)$ & $\mathrm{pc}$ & \\
\hline 16 & 49 & 877.3177 & 113983.79 & 877.3155 & 0.0020 & -0.29 & 0.0022 & 8 & 11 & $5 d^{2} 6 s\left({ }^{3} F\right)^{4} F_{7 / 2}$ & 191690.18 & $305674.26(7 / 2)$ & $\mathrm{pc}$ & \\
\hline 31 & 193 & 917.2159 & 109025.59 & 917.2149 & 0.0021 & -0.12 & 0.0010 & 8 & 12 & $5 d^{2} 6 s\left({ }^{3} F\right)^{2} F_{5 / 2}$ & 196648.56 & $305674.26(7 / 2)$ & $\mathrm{pc}$ & \\
\hline 20 & 24 & 929.0956 & 107631.55 & 929.0968 & 0.0050 & 0.14 & -0.0012 & 9 & 10 & $5 d^{2} 6 s\left({ }^{1} D\right)^{2} D_{3 / 2}$ & 208080.78 & $315712.19(5 / 2)$ & $\mathrm{pc} *$ & \\
\hline 42 & 345 & 948.8863 & 105386.70 & 948.8840 & 0.0024 & -0.26 & 0.0023 & 9 & 10 & $5 d^{2} 6 s\left({ }^{3} F\right){ }^{4} F_{5 / 2}$ & 179664.83 & $285051.79(7 / 2)$ & $\mathrm{pd}$ & \\
\hline 14 & 97 & 957.2701 & 104463.73 & 957.2727 & 0.0021 & 0.29 & -0.0026 & 13 & 11 & $5 d^{2} 6 s\left({ }^{3} F\right)^{4} F_{7 / 2}$ & 191690.18 & $296153.62(5 / 2)$ & $\mathrm{pd}$ & \\
\hline 22 & 165 & 963.5136 & 103786.81 & 963.5158 & 0.0024 & 0.23 & -0.0022 & 7 & 11 & $5 d^{2} 6 s\left({ }^{3} F\right)^{4} F_{7 / 2}$ & 191690.18 & $295476.76(9 / 2)$ & pd & \\
\hline
\end{tabular}




\begin{tabular}{|c|c|c|c|c|c|c|c|c|c|c|c|c|c|c|}
\hline 12 & 159 & 964.4045 & 103690.93 & 964.4056 & 0.0023 & 0.12 & -0.0011 & 10 & 10 & $5 d^{2} 6 s\left({ }^{3} F\right)^{2} F_{7 / 2}$ & 208463.13 & $312153.94(9 / 2)$ & $\mathrm{pd}$ & 100 \\
\hline 17 & 69 & 971.2240 & 102962.86 & 971.2217 & 0.0030 & -0.24 & 0.0023 & 12 & 10 & $5 d^{2} 6 s\left({ }^{3} F\right)^{2} F_{7 / 2}$ & 208463.13 & $311426.23(7 / 2)$ & pd & \\
\hline 5 & 41 & 987.1073 & 101306.11 & 987.1118 & 0.0040 & 0.46 & -0.0045 & 13 & 9 & $5 d^{2} 6 s\left({ }^{3} P\right)^{4} P_{3 / 2}$ & 202719.09 & $304024.73(5 / 2)$ & pd* & \\
\hline 17 & 68 & 992.7225 & 100733.09 & 992.7206 & 0.0030 & -0.19 & 0.0019 & 13 & 9 & $5 d^{2} 6 s\left({ }^{3} P\right){ }^{4} P_{5 / 2}$ & 203291.46 & $304024.73(5 / 2)$ & pd & \\
\hline 21 & 62 & 1003.7365 & 99627.74 & 1003.7336 & 0.0030 & -0.28 & 0.0029 & 7 & 6 & $5 d^{2} 6 s\left({ }^{1} G\right)^{2} G_{9 / 2}$ & 218855.29 & $318483.31(9 / 2)$ & pd & \\
\hline 8 & 85 & 1004.9758 & 99504.88 & 1004.9740 & 0.0023 & -0.18 & 0.0018 & 13 & 12 & $5 d^{2} 6 s\left({ }^{3} F\right)^{2} F_{5 / 2}$ & 196648.56 & $296153.62(5 / 2)$ & pd & \\
\hline 24 & 491 & 1022.9397 & 97757.47 & 1022.9468 & 0.0031 & 0.68 & -0.0071 & 7 & 7 & $5 d^{2} 6 s\left({ }^{3} F\right)^{4} F_{3 / 2}$ & 176039.47 & $273796.26(3 / 2)$ & pd & 300 \\
\hline 30 & 451 & 1027.6000 & 97314.13 & 1027.6020 & 0.0030 & 0.19 & -0.0020 & 11 & 9 & $5 d^{2} 6 s\left({ }^{3} P\right){ }^{4} P_{3 / 2}$ & 202719.09 & $300033.03(3 / 2)$ & pd & 200 \\
\hline 122 & 512 & 1032.2468 & 96876.06 & 1032.2503 & 0.0030 & 0.33 & -0.0035 & 10 & 7 & $5 d^{2} 6 s\left({ }^{3} F\right)^{4} F_{9 / 2}$ & 200756.58 & $297632.31(7 / 2)$ & $\mathrm{pd}$ & \\
\hline 35 & 371 & 1036.0592 & 96519.58 & 1036.0686 & 0.0040 & 0.88 & -0.0094 & 7 & 8 & $5 d^{2} 6 s\left({ }^{1} G\right)^{2} G_{7 / 2}$ & 221964.61 & $318483.31(9 / 2)$ & $\mathrm{pd} *$ & 200, Pt V \\
\hline 82 & 349 & 1055.0544 & 94781.84 & 1055.0599 & 0.0030 & 0.50 & -0.0055 & 8 & 11 & $5 d^{2} 6 s\left({ }^{3} F\right)^{4} F_{7 / 2}$ & 191690.18 & $286471.53(5 / 2)$ & pd & \\
\hline 114 & 487 & 1055.7417 & 94720.14 & 1055.7413 & 0.0030 & -0.04 & 0.0004 & 7 & 7 & $5 d^{2} 6 s\left({ }^{3} F\right)^{4} F_{9 / 2}$ & 200756.58 & $295476.76(9 / 2)$ & pd & \\
\hline 41 & 205 & 1060.9904 & 94251.56 & 1060.9905 & 0.0040 & 0.01 & -0.0001 & 9 & 6 & $5 d^{2} 6 s\left({ }^{1} D\right)^{2} D_{5 / 2}$ & 221460.64 & $315712.19(5 / 2)$ & pd & \\
\hline 61 & 333 & 1062.3515 & 94130.80 & 1062.3444 & 0.0030 & -0.63 & 0.0071 & 7 & 10 & $5 d^{2} 6 s\left({ }^{3} F\right)^{4} F_{5 / 2}$ & 179664.83 & $273796.26(3 / 2)$ & pd & \\
\hline 70 & 175 & 1068.7680 & 93565.68 & 1068.7685 & 0.0040 & 0.04 & -0.0005 & 6 & 6 & $5 d^{2} 6 s\left({ }^{1} D\right)^{2} D_{5 / 2}$ & 221460.64 & $315026.28(3 / 2)$ & pd & \\
\hline 26 & 254 & 1070.2684 & 93434.51 & 1070.2681 & 0.0030 & -0.03 & 0.0003 & 13 & 9 & $5 d^{2} 6 s\left({ }^{3} \mathrm{P}\right){ }^{4} \mathrm{P}_{3 / 2}$ & 202719.09 & $296153.62(5 / 2)$ & pd & \\
\hline 75 & 349 & 1071.1067 & 93361.38 & 1071.1041 & 0.0030 & -0.23 & 0.0026 & 9 & 11 & $5 d^{2} 6 s\left({ }^{3} F\right)^{4} F_{7 / 2}$ & 191690.18 & $285051.79(7 / 2)$ & pd & \\
\hline 91 & 331 & 1071.8342 & 93298.01 & 1071.8269 & 0.0030 & -0.64 & 0.0073 & 10 & 6 & $5 d^{2} 6 s\left({ }^{1} G\right)^{2} G_{9 / 2}$ & 218855.29 & $312153.94(9 / 2)$ & pd & \\
\hline 18 & 88 & 1074.8246 & 93038.44 & 1074.8250 & 0.0030 & 0.03 & -0.0004 & 7 & 9 & $5 d^{2} 6 s\left({ }^{3} \mathrm{P}\right)^{4} \mathrm{P}_{3 / 2}$ & 202719.09 & $295757.49(1 / 2)$ & $\mathrm{pd}$ & \\
\hline 40 & 341 & 1076.8674 & 92861.94 & 1076.8648 & 0.0030 & -0.22 & 0.0026 & 13 & 9 & $5 d^{2} 6 s\left({ }^{3} P\right){ }^{4} P_{5 / 2}$ & 203291.46 & $296153.62(5 / 2)$ & pd & \\
\hline 84 & 475 & 1080.2485 & 92571.29 & 1080.2526 & 0.0030 & 0.35 & -0.0041 & 12 & 6 & $5 d^{2} 6 s\left({ }^{1} G\right)^{2} G_{9 / 2}$ & 218855.29 & $311426.23(7 / 2)$ & $\mathrm{pd}$ & $300, \mathrm{Pt} \mathrm{V}$ \\
\hline 17 & 41 & 1087.5237 & 91952.02 & 1087.5210 & 0.0030 & -0.23 & 0.0027 & 11 & 10 & $5 d^{2} 6 s\left({ }^{1} D\right)^{2} D_{3 / 2}$ & 208080.78 & $300033.03(3 / 2)$ & $\mathrm{pd}$ & \\
\hline 70 & 609 & 1090.4921 & 91701.72 & 1090.4896 & 0.0030 & -0.21 & 0.0025 & 6 & 7 & $5 d^{2} 6 s\left({ }^{3} F\right)^{4} F 3_{/ 2}$ & 176039.47 & $267741.40(5 / 2)$ & pd & \\
\hline 18 & 186 & 1091.1139 & 91649.46 & 1091.1215 & 0.0050 & 0.64 & -0.0076 & 9 & 9 & $5 d^{2} 6 s\left({ }^{3} \mathrm{P}\right){ }^{4} \mathrm{P} 3_{/ 2}$ & 202719.09 & $294367.91(3 / 2)$ & $\mathrm{pd} *$ & ${ }^{*} 100,2 \mathrm{~d}$ ord \\
\hline 34 & 89 & 1092.3859 & 91542.74 & 1092.3855 & 0.0040 & -0.03 & 0.0004 & 4 & 4 & $5 d^{2} 6 s\left({ }^{3} \mathrm{P}\right){ }^{4} \mathrm{P} 1_{/ 2}$ & 195671.13 & $287213.90(1 / 2)$ & $\mathrm{pd}$ & \\
\hline 44 & 252 & 1097.9764 & 91076.64 & 1097.9786 & 0.0040 & 0.19 & -0.0022 & 9 & 9 & $5 d^{2} 6 s\left({ }^{3} \mathrm{P}\right)^{4} \mathrm{P} 5_{/ 2}$ & 203291.46 & $294367.91(3 / 2)$ & pd & Pt VI(100) \\
\hline 62 & 177 & 1108.7721 & 90189.86 & 1108.7787 & 0.0030 & 0.53 & -0.0066 & 10 & 8 & $5 d^{2} 6 s\left({ }^{1} G\right)^{2} G_{7 / 2}$ & 221964.61 & $312153.94(9 / 2)$ & $\mathrm{pd}$ & \\
\hline 30 & 108 & 1113.3060 & 89822.57 & 1113.3009 & 0.0030 & -0.41 & 0.0050 & 8 & 12 & $5 d^{2} 6 s\left({ }^{3} F\right)^{2} F_{5 / 2}$ & 196648.56 & $286471.53(5 / 2)$ & pd & \\
\hline 49 & 127 & 1117.8024 & 89461.25 & 1117.7978 & 0.0030 & -0.37 & 0.0046 & 12 & 8 & $5 d^{2} 6 s\left({ }^{1} G\right)^{2} G_{7 / 2}$ & 221964.61 & $311426.23(7 / 2)$ & $\mathrm{pd}$ & \\
\hline 47 & 214 & 1121.4663 & 89168.98 & 1121.4637 & 0.0030 & -0.21 & 0.0026 & 10 & 10 & $5 d^{2} 6 s\left({ }^{3} F\right)^{2} F_{7 / 2}$ & 208463.13 & $297632.31(7 / 2)$ & pd & Pt IV \\
\hline 52 & 216 & 1131.1778 & 88403.43 & 1131.1803 & 0.0030 & 0.20 & -0.0025 & 9 & 12 & $5 d^{2} 6 s\left({ }^{3} F\right)^{2} F_{5 / 2}$ & 196648.56 & $285051.79(7 / 2)$ & pd & \\
\hline 43 & 856 & 1135.3755 & 88076.59 & 1135.3757 & 0.0030 & 0.01 & -0.0002 & 6 & 10 & $5 d^{2} 6 s\left({ }^{3} F\right)^{4} F_{5 / 2}$ & 179664.83 & $267741.40(5 / 2)$ & $\mathrm{pd}$ & $300,2 \mathrm{~d}$ ord \\
\hline 11 & 68 & 1140.5561 & 87676.53 & 1140.5537 & 0.0030 & -0.18 & 0.0024 & 7 & 10 & $5 d^{2} 6 s\left({ }^{1} D\right)^{2} D_{3 / 2}$ & 208080.78 & $295757.49(1 / 2)$ & $\mathrm{pd}$ & Pt VII \\
\hline 72 & 210 & 1149.2472 & 87013.48 & 1149.2453 & 0.0030 & -0.15 & 0.0019 & 7 & 10 & $5 d^{2} 6 s\left({ }^{3} F\right)^{2} F_{7 / 2}$ & 208463.13 & $295476.76(9 / 2)$ & $\mathrm{pd}$ & \\
\hline
\end{tabular}


Table 2

Experimental and calculated energy levels $\left(\mathrm{cm}^{-1}\right)$ in the $5 \mathrm{~d}^{3}$ and $5 \mathrm{~d}^{2} 6 \mathrm{~s}$ configurations of Pt VIII.

\begin{tabular}{|c|c|c|c|c|c|c|c|c|c|c|}
\hline $\mathrm{E}_{\mathrm{obs}}$ & $\mathrm{E}_{\text {calc }}$ & $\Delta$ & $\mathrm{N}$ & $\mathrm{N}_{\mathrm{opt}}$ & $\operatorname{rwmv}_{1}$ & $\mathrm{rwmv}_{2}$ & Unc & Composition & & \\
\hline \multicolumn{11}{|l|}{$\mathrm{J}=1 / 2$} \\
\hline- & 250250.38 & - & & & & & & $88 \% 2 \mid\left({ }^{1} S\right){ }^{2} \mathrm{~S}$ & $+\quad 6 \% 2\left({ }^{3} \mathrm{P}\right){ }^{4} \mathrm{P}$ & $+\quad 6 \% 2\left({ }^{3} \mathrm{P}\right){ }^{2} \mathrm{P}$ \\
\hline 213105.19 & 213090.63 & 14.56 & 4 & 4 & 0.22 & 0.20 & 0.40 & $88 \% 2 \mid\left({ }^{3} \mathrm{P}\right){ }^{2} \mathrm{P}$ & $+\quad 9 \% 2\left({ }^{3} \mathrm{P}\right){ }^{4} \mathrm{P}$ & $+\quad 3 \% 2 \mid\left({ }^{1} \mathrm{~S}\right){ }^{2} \mathrm{~S}$ \\
\hline 195671.13 & 195669.43 & 1.70 & 4 & 4 & 0.22 & 0.11 & 0.40 & $84 \% 2 \mid\left({ }^{3} \mathrm{P}\right){ }^{4} \mathrm{P}$ & $+10 \% 2\left({ }^{1} \mathrm{~S}\right){ }^{2} \mathrm{~S}$ & $+\quad 6 \% 2\left({ }^{3} \mathrm{P}\right){ }^{2} \mathrm{P}$ \\
\hline 39855.71 & 39868.50 & -12.79 & 9 & 7 & 0.90 & 0.73 & 1.10 & $\left.68 \% 1\right|^{2} \mathrm{P}$ & $+\left.32 \% 1\right|^{4} \mathrm{P}$ & \\
\hline 22003.95 & 22005.11 & -1.16 & 9 & 8 & 0.80 & 0.72 & 1.00 & $\left.68 \% 1\right|^{4} \mathrm{P}$ & $+\left.32 \% 1\right|^{2} \mathrm{P}$ & \\
\hline \multicolumn{11}{|c|}{ 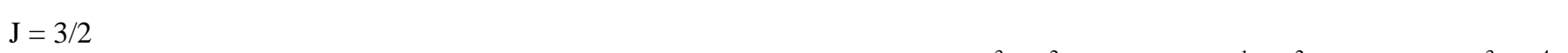 } \\
\hline- & 227605.79 & - & & & & & & $67 \% 2 \mid\left({ }^{3} \mathrm{P}\right){ }^{2} \mathrm{P}$ & $+27 \% 2 \mid\left({ }^{1} \mathrm{D}\right){ }^{2} \mathrm{D}$ & $+\quad 3 \% 2\left({ }^{3} \mathrm{P}\right){ }^{4} \mathrm{P}$ \\
\hline 208080.78 & 208101.17 & -20.39 & 10 & 9 & 0.14 & 0.11 & 0.30 & $49 \% 2 \mid\left({ }^{1} \mathrm{D}\right){ }^{2} \mathrm{D}$ & $+31 \% 2\left({ }^{3} \mathrm{P}\right){ }^{2} \mathrm{P}$ & $+13 \% 2\left({ }^{3} \mathrm{P}\right){ }^{4} \mathrm{P}$ \\
\hline 202719.09 & 202716.08 & 3.01 & 9 & 5 & 0.18 & 0.09 & 0.30 & $84 \% 2 \mid\left({ }^{3} \mathrm{P}\right){ }^{4} \mathrm{P}$ & $+13 \% 2 \mid\left({ }^{1} \mathrm{D}\right){ }^{2} \mathrm{D}$ & $+\quad 3 \% 2\left({ }^{3} \mathrm{~F}\right){ }^{4} \mathrm{~F}$ \\
\hline 176039.47 & 176029.49 & 9.98 & 7 & 7 & 0.17 & 0.20 & 0.30 & $89 \% 2\left({ }^{3} \mathrm{~F}\right){ }^{4} \mathrm{~F}$ & $+11 \% 2\left({ }^{1} \mathrm{D}\right){ }^{2} \mathrm{D}$ & \\
\hline 75993.15 & 75997.41 & -4.26 & 10 & 9 & 0.72 & 0.41 & 0.80 & $\left.62 \% 1\right|^{2} \mathrm{D} 1$ & $+\left.36 \% 1\right|^{2} \mathrm{D} 2$ & $+\left.\quad 1 \% 1\right|^{2} \mathrm{P}$ \\
\hline 56003.81 & 56002.74 & 1.07 & 15 & 12 & 0.67 & 0.49 & 0.90 & $\left.56 \% 1\right|^{2} \mathrm{P}$ & $+\left.18 \% 1\right|^{2} \mathrm{D} 1$ & $+\left.13 \% 1\right|^{2} \mathrm{D} 2$ \\
\hline 38152.74 & 38151.15 & 1.59 & 16 & 15 & 0.58 & 0.37 & 0.70 & $\left.34 \% 1\right|^{2} \mathrm{D} 2$ & $+\left.41 \% 1\right|^{4} \mathrm{P}$ & $+\left.10 \% 1\right|^{2} \mathrm{D} 1$ \\
\hline 18763.68 & 18757.02 & 6.66 & 14 & 14 & 0.62 & 0.50 & 0.80 & $46 \% 1{ }^{4} \mathrm{P}$ & $+\left.33 \% 1\right|^{2} \mathrm{P}$ & $+\left.13 \% 1\right|^{4} \mathrm{~F}$ \\
\hline 0.00 & 7.89 & -7.89 & 12 & 10 & 0.82 & 0.56 & 1.00 & $\left.76 \% 1\right|^{4} \mathrm{~F}$ & $+\left.12 \% 1\right|^{2} \mathrm{D} 2$ & $+\left.\quad 8 \% 1\right|^{2} \mathrm{D} 1$ \\
\hline \multicolumn{11}{|l|}{$\mathrm{J}=5 / 2$} \\
\hline 221460.64 & 221442.00 & 18.64 & 6 & 5 & 0.19 & 0.04 & 0.40 & $53 \% 2 \mid\left({ }^{1} \mathrm{D}\right){ }^{2} \mathrm{D}$ & $+41 \% 2 \mid\left({ }^{3} \mathrm{P}\right) 4 \mathrm{P}$ & $+\quad 4 \% 2\left({ }^{3} \mathrm{~F}\right){ }^{2} \mathrm{~F}$ \\
\hline 203291.46 & 203307.64 & -16.18 & 9 & 8 & 0.15 & 0.12 & 0.20 & $51 \% 2 \mid\left({ }^{3} \mathrm{P}\right){ }^{4} \mathrm{P}$ & $+27 \% 2\left({ }^{3} \mathrm{~F}\right) 2 \mathrm{~F}$ & $+22 \% 22\left({ }^{1} \mathrm{D}\right){ }^{2} \mathrm{D}$ \\
\hline 196648.56 & 196649.17 & -0.61 & 12 & 10 & 0.13 & 0.10 & 0.20 & $50 \% 2 \mid\left({ }^{3} \mathrm{~F}\right){ }^{2} \mathrm{~F}$ & $+31 \% 2\left({ }^{3} \mathrm{~F}\right) 4 \mathrm{~F}$ & $+12 \% 2\left({ }^{1} \mathrm{D}\right){ }^{2} \mathrm{D}$ \\
\hline 179664.83 & 179671.85 & -7.02 & 10 & 8 & 0.15 & 0.15 & 0.30 & $67 \% 2 \mid\left({ }^{3} \mathrm{~F}\right){ }^{4} \mathrm{~F}$ & $+18 \% 2\left({ }^{3} \mathrm{~F}\right) 2 \mathrm{~F}$ & $+13 \% 2\left({ }^{1} \mathrm{D}\right){ }^{2} \mathrm{D}$ \\
\hline 78845.26 & 78842.87 & 2.39 & 7 & 7 & 0.77 & 0.43 & 0.90 & $\left.76 \% 1\right|^{2} \mathrm{D} 1$ & $+\left.15 \% 1\right|^{2} \mathrm{~F}$ & $+\quad 5 \% 1{ }^{4} \mathrm{P}$ \\
\hline 50624.30 & 50622.75 & 1.55 & 16 & 15 & 0.56 & 0.45 & 0.70 & $\left.82 \% 1\right|^{2} \mathrm{~F}$ & $+\left.11 \% 1\right|^{2} \mathrm{D} 1$ & $+\quad 6 \% 1{ }^{4} \mathrm{P}$ \\
\hline 46366.31 & 46368.88 & -2.57 & 18 & 18 & 0.53 & 0.41 & 0.70 & $\left.89 \% 1\right|^{2} \mathrm{D} 2$ & $+\quad 5 \% 1{ }^{4} \mathrm{~F}$ & $+\quad 3 \% 1{ }^{4} \mathrm{P}$ \\
\hline 33389.80 & 33391.55 & -1.75 & 17 & 15 & 0.62 & 0.46 & 0.80 & $\left.86 \% 1\right|^{4} \mathrm{P}$ & $+\left.\quad 9 \% 1\right|^{2} \mathrm{D} 1$ & $+\left.\quad 2 \% 1\right|^{2} \mathrm{~F}$ \\
\hline 10194.49 & 10193.12 & 1.37 & 16 & 15 & 0.67 & 0.53 & 0.80 & $\left.93 \% 1\right|^{4} \mathrm{~F}$ & $+\left.5 \% 1\right|^{2} \mathrm{D} 2$ & $+\left.\quad 2 \% 1\right|^{2} \mathrm{D} 1$ \\
\hline \multicolumn{11}{|l|}{$\mathrm{J}=7 / 2$} \\
\hline 221964.61 & 221983.07 & -18.46 & 8 & 7 & 0.15 & 0.16 & 0.30 & $55 \% 2 \mid\left({ }^{1} \mathrm{G}\right){ }^{2} \mathrm{G}$ & $+40 \% 2\left({ }^{3} \mathrm{~F}\right){ }^{2} \mathrm{~F}$ & $+\quad 5 \% 2\left({ }^{3} \mathrm{~F}\right){ }^{4} \mathrm{~F}$ \\
\hline 208463.13 & 208442.12 & 21.01 & 10 & 10 & 0.13 & 0.12 & 0.23 & $53 \% 2 \mid\left({ }^{3} \mathrm{~F}\right){ }^{2} \mathrm{~F}$ & $+44 \% 2 \mid\left({ }^{1} \mathrm{G}\right){ }^{2} \mathrm{G}$ & $+\quad 2 \% 2\left({ }^{3} \mathrm{~F}\right){ }^{4} \mathrm{~F}$ \\
\hline 191690.18 & 191690.53 & -0.35 & 11 & 11 & 0.13 & 0.12 & 0.00 & $93 \% 2 \mid\left({ }^{3} \mathrm{~F}\right){ }^{4} \mathrm{~F}$ & $+\quad 7 \% 2\left({ }^{3} \mathrm{~F}\right){ }^{2} \mathrm{~F}$ & $+\quad 1 \% 2\left({ }^{1} \mathrm{G}\right){ }^{2} \mathrm{C}$ \\
\hline 52580.76 & 52578.76 & 2.00 & 14 & 13 & 0.60 & 0.27 & 0.70 & $\left.93 \% 1\right|^{2} \mathrm{~F}$ & $+\left.7 \% 1\right|^{2} \mathrm{G}$ & $+\quad 1 \% 1{ }^{4} \mathrm{~F}$ \\
\hline 31715.83 & 31711.40 & 4.43 & 16 & 14 & 0.65 & 0.57 & 0.80 & $\left.73 \% 1\right|^{2} \mathrm{G}$ & $+\left.23 \% 1\right|^{4} \mathrm{~F}$ & $+\left.4 \% 1\right|^{2} \mathrm{~F}$ \\
\hline 17083.81 & 17079.47 & 4.34 & 15 & 15 & 0.65 & 0.59 & 0.90 & $\left.76 \% 1\right|^{4} \mathrm{~F}$ & $+\left.20 \% 1\right|^{2} \mathrm{G}$ & $+\quad 4 \% 1{ }^{2} \mathrm{~F}$ \\
\hline \multicolumn{11}{|l|}{$J=9 / 2$} \\
\hline 218855.29 & 218854.88 & 0.41 & 6 & 6 & 0.17 & 0.19 & 0.30 & $82 \% 2 \mid\left({ }^{1} G\right){ }^{2} G$ & $+18 \% 2 \mid\left({ }^{3} \mathrm{~F}\right){ }^{4} \mathrm{~F}$ & \\
\hline 200756.58 & 200755.93 & 0.65 & 7 & 7 & 0.16 & 0.11 & 0.30 & $82 \% 2 \mid\left({ }^{3} \mathrm{~F}\right){ }^{4} \mathrm{~F}$ & $+18 \% 2 \mid\left({ }^{1} \mathrm{G}\right){ }^{2} \mathrm{G}$ & \\
\hline 51970.45 & 51971.51 & -1.06 & 9 & 9 & 0.75 & 0.55 & 0.90 & $\left.58 \% 1\right|^{2} \mathrm{G}$ & $+\left.27 \% 1\right|^{2} \mathrm{H}$ & $+\left.15 \% 1\right|^{4} \mathrm{~F}$ \\
\hline 33428.03 & 33430.40 & -2.37 & 12 & 12 & 0.71 & 0.54 & 0.90 & $\left.53 \% 1\right|^{2} \mathrm{H}$ & $+44 \% 1{ }^{4} \mathrm{~F}$ & $+\left.\quad 3 \% 1\right|^{2} \mathrm{G}$ \\
\hline 20563.94 & 20565.47 & -1.53 & 11 & 11 & 0.76 & 0.70 & 1.00 & $\left.41 \% 1\right|^{4} \mathrm{~F}$ & $+\left.39 \% 1\right|^{2} \mathrm{G}$ & $+\left.20 \% 1\right|^{2} \mathrm{H}$ \\
\hline \multicolumn{11}{|l|}{$\mathrm{J}=11 / 2$} \\
\hline 43289.57 & 43289.85 & -0.28 & 7 & 6 & 0.93 & 0.58 & 1.10 & $\left.100 \% 1\right|^{2} \mathrm{H}$ & & \\
\hline
\end{tabular}


Table 3

Experimental and calculated energy levels $\left(\mathrm{cm}^{-1}\right)$ in the $5 \mathrm{~d}^{2} 6 \mathrm{p}$ configuration of Pt VIII.

\begin{tabular}{|c|c|c|c|c|c|c|c|c|c|c|}
\hline $\mathrm{E}_{\mathrm{obs}}$ & $\mathrm{E}_{\text {calc }}$ & $\Delta$ & $\mathrm{N}$ & $\mathrm{N}_{\mathrm{opt}}$ & rwmv $_{1}$ & rwmv $_{2}$ & $2 \mathrm{Unc}$ & \multicolumn{2}{|l|}{ Composition } & \\
\hline \multicolumn{11}{|l|}{$\mathbf{J}=1 / 2$} \\
\hline 339368.34 & 339169.35 & 198.99 & 6 & 5 & 0.34 & 0.41 & 0.40 & $48 \% \quad\left({ }^{3} \mathrm{P}\right)^{2} \mathrm{P}$ & $+24 \%\left({ }^{1} \mathrm{~S}\right)^{2} \mathrm{P}$ & $+16 \%\left({ }^{3} \mathrm{P}\right)^{4} \mathrm{D}$ \\
\hline 336229.51 & 336406.09 & -176.58 & 4 & 4 & 0.33 & 0.27 & 0.40 & $30 \% \quad\left({ }^{1} \mathrm{D}\right)^{2} \mathrm{P}$ & $+27 \%\left({ }^{1} \mathrm{~S}\right)^{2} \mathrm{P}$ & $+21 \%\left({ }^{3} \mathrm{P}\right)^{2} \mathrm{~S}$ \\
\hline 325884.46 & 325832.51 & 51.95 & 4 & 4 & 0.30 & 0.06 & 0.40 & $39 \% \quad\left({ }^{3} \mathrm{P}\right)^{4} \mathrm{P}$ & $+26 \%\left({ }^{1} \mathrm{D}\right)^{2} \mathrm{P}$ & $+22 \%\left({ }^{3} \mathrm{P}\right)^{2} \mathrm{~S}$ \\
\hline 287213.90 & 286984.51 & 229.39 & 4 & 4 & 0.32 & 0.49 & 0.50 & $48 \% \quad\left({ }^{3} \mathrm{P}\right)^{4} \mathrm{D}$ & $+17 \%\left({ }^{3} \mathrm{P}\right)^{2} \mathrm{~S}$ & $+12 \%\left({ }^{1} \mathrm{~S}\right)^{2} \mathrm{P}$ \\
\hline \multicolumn{11}{|l|}{$\mathrm{J}=3 / 2$} \\
\hline 352661.04 & 353060.90 & -399.86 & 6 & 6 & 1.06 & 0.49 & 1.20 & $45 \% \quad\left({ }^{3} \mathrm{P}\right)^{2} \mathrm{P}$ & $+19 \%\left({ }^{1} \mathrm{D}\right)^{2} \mathrm{D}$ & $+12 \%\left({ }^{3} \mathrm{P}\right)^{2} \mathrm{D}$ \\
\hline 375831.17 & 375819.94 & 11.23 & 4 & 4 & 1.44 & 0.50 & 1.50 & $86 \% \quad\left({ }^{1} \mathrm{~S}\right)^{2} \mathrm{P}$ & $+\quad 4 \%\left({ }^{3} \mathrm{P}\right){ }^{4} \mathrm{P}$ & $+\quad 3 \%\left({ }^{3} \mathrm{P}\right)^{2} \mathrm{D}$ \\
\hline 315026.28 & 314990.70 & 35.58 & 6 & 6 & 0.33 & 0.37 & 0.50 & $31 \% \quad\left({ }^{3} \mathrm{P}\right)^{4} \mathrm{~S}$ & $+24 \%\left({ }^{1} \mathrm{D}\right)^{2} \mathrm{P}$ & $+20 \%\left({ }^{1} \mathrm{D}\right)^{2} \mathrm{D}$ \\
\hline 302624.91 & 302665.82 & -40.91 & 5 & 5 & 0.32 & 0.45 & 0.50 & $32 \% \quad\left({ }^{3} \mathrm{~F}\right)^{4} \mathrm{~F}$ & $+29 \%\left({ }^{3} \mathrm{~F}\right)^{4} \mathrm{D}$ & $+16 \%\left({ }^{3} \mathrm{~F}\right)^{2} \mathrm{D}$ \\
\hline 300033.03 & 300274.60 & -241.57 & 11 & 9 & 0.24 & 0.21 & 0.40 & $36 \% \quad\left({ }^{3} \mathrm{P}\right)^{4} \mathrm{D}$ & $+25 \%\left({ }^{3} \mathrm{P}\right)^{4} \mathrm{~S}$ & $+15 \%\left({ }^{3} \mathrm{P}\right)^{2} \mathrm{P}$ \\
\hline 294367.91 & 294329.40 & 38.51 & 9 & 7 & 0.31 & 0.47 & 0.40 & $31 \% \quad\left({ }^{3} \mathrm{P}\right)^{4} \mathrm{D}$ & $+18 \%\left({ }^{1} \mathrm{D}\right)^{2} \mathrm{P}$ & $+14 \%\left({ }^{1} \mathrm{D}\right)^{2} \mathrm{D}$ \\
\hline 273796.26 & 273760.23 & 36.03 & 7 & 7 & 0.25 & 0.42 & 0.50 & $46 \% \quad\left({ }^{3} \mathrm{~F}\right)^{4} \mathrm{~F}$ & $+31 \%\left({ }^{3} \mathrm{~F}\right)^{2} \mathrm{D}$ & $+\quad 9 \%\left({ }^{3} \mathrm{~F}\right)^{4} \mathrm{D}$ \\
\hline \multicolumn{11}{|l|}{$\mathrm{J}=5 / 2$} \\
\hline 354205.09 & 354034.13 & 170.96 & 8 & 8 & 0.35 & 0.28 & 0.50 & $45 \% \quad\left({ }^{1} \mathrm{G}\right)^{2} \mathrm{~F}$ & $+19 \%\left({ }^{3} \mathrm{~F}\right)^{2} \mathrm{D}$ & $+11 \%\left({ }^{3} \mathrm{P}\right)^{2} \mathrm{D}$ \\
\hline 347359.74 & 347317.51 & 42.23 & 10 & 10 & 0.28 & 0.14 & 0.40 & $38 \% \quad\left({ }^{1} \mathrm{G}\right)^{2} \mathrm{~F}$ & $+26 \%\left({ }^{3} \mathrm{P}\right)^{4} \mathrm{P}$ & $+19 \%\left({ }^{1} \mathrm{D}\right)^{2} \mathrm{D}$ \\
\hline 337170.08 & 337273.17 & -103.09 & 9 & 9 & 0.33 & 0.25 & 0.40 & $31 \% \quad\left({ }^{3} \mathrm{~F}\right)^{2} \mathrm{D}$ & $+19 \%\left({ }^{1} \mathrm{D}\right)^{2} \mathrm{D}$ & $+17 \%\left({ }^{3} \mathrm{P}\right)^{2} \mathrm{D}$ \\
\hline 267741.40 & 267943.63 & -202.23 & 6 & 6 & 0.22 & 0.30 & 0.30 & $60 \% \quad\left({ }^{3} \mathrm{~F}\right)^{4} \mathrm{G}$ & $+19 \%\left({ }^{3} \mathrm{~F}\right)^{2} \mathrm{~F}$ & $+13 \%\left({ }^{1} \mathrm{D}\right)^{2} \mathrm{~F}$ \\
\hline \multicolumn{11}{|l|}{$\mathrm{J}=7 / 2$} \\
\hline 348029.10 & 348051.68 & -22.58 & 9 & 8 & 0.33 & 0.20 & 0.40 & $31 \% \quad\left({ }^{1} \mathrm{G}\right)^{2} \mathrm{~F}$ & $+21 \%\left({ }^{3} \mathrm{P}\right)^{4} \mathrm{D}$ & $+19 \%\left({ }^{1} \mathrm{D}\right)^{2} \mathrm{~F}$ \\
\hline 345243.77 & 345125.72 & 118.05 & 6 & 6 & 0.31 & 0.35 & 0.40 & $30 \% \quad\left({ }^{1} \mathrm{D}\right)^{2} \mathrm{~F}$ & $+23 \%\left({ }^{3} \mathrm{P}\right)^{4} \mathrm{D}$ & $+12 \%\left({ }^{1} \mathrm{G}\right)^{2} \mathrm{G}$ \\
\hline 332147.94 & 332082.44 & 65.50 & 11 & 10 & 0.27 & 0.33 & 0.50 & $27 \% \quad\left({ }^{3} \mathrm{P}\right)^{4} \mathrm{D}$ & $+17 \%\left({ }^{1} \mathrm{G}\right)^{2} \mathrm{~F}$ & $+16 \%\left({ }^{3} \mathrm{~F}\right)^{2} \mathrm{~F}$ \\
\hline 324098.89 & 324094.06 & 4.83 & 7 & 7 & 0.22 & 0.28 & 0.40 & $25 \% \quad\left({ }^{3} \mathrm{P}\right)^{4} \mathrm{D}$ & $+16 \%\left({ }^{3} \mathrm{~F}\right)^{4} \mathrm{D}$ & $+15 \%\left({ }^{3} \mathrm{~F}\right)^{2} \mathrm{~F}$ \\
\hline 319124.12 & 319090.38 & 33.74 & 10 & 10 & 0.31 & 0.25 & 0.40 & $32 \% \quad\left({ }^{3} \mathrm{~F}\right)^{4} \mathrm{~F}$ & $+28 \%\left({ }^{3} \mathrm{~F}\right)^{2} \mathrm{G}$ & $+14 \%\left({ }^{3} \mathrm{~F}\right)^{2} \mathrm{~F}$ \\
\hline 311426.23 & 311467.38 & -41.15 & 12 & 11 & 0.20 & 0.19 & 0.30 & $45 \% \quad\left({ }^{1} G\right)^{2} G$ & $+24 \%\left({ }^{1} \mathrm{G}\right)^{2} \mathrm{~F}$ & $+20 \%\left({ }^{3} \mathrm{~F}\right)^{4} \mathrm{~F}$ \\
\hline 305674.26 & 305566.20 & 108.06 & 8 & 8 & 0.22 & 0.24 & 0.30 & $37 \% \quad\left({ }^{3} \mathrm{~F}\right)^{2} \mathrm{G}$ & $+20 \%\left({ }^{3} \mathrm{~F}\right)^{4} \mathrm{G}$ & $+13 \%\left({ }^{1} \mathrm{D}\right)^{2} \mathrm{~F}$ \\
\hline 297632.31 & 297607.00 & 25.31 & 10 & 8 & 0.21 & 0.16 & 0.30 & $35 \% \quad\left({ }^{3} \mathrm{~F}\right)^{4} \mathrm{D}$ & $+22 \%\left({ }^{3} \mathrm{~F}\right)^{4} \mathrm{~F}$ & $+16 \%\left({ }^{1} \mathrm{G}\right)^{2} \mathrm{G}$ \\
\hline 285051.79 & 285074.34 & -22.55 & 9 & 9 & 0.20 & 0.16 & 0.24 & $68 \% \quad\left({ }^{3} \mathrm{~F}\right)^{4} \mathrm{G}$ & $+12 \%\left({ }^{3} \mathrm{~F}\right)^{2} \mathrm{~F}$ & $+10 \%\left({ }^{3} \mathrm{~F}\right)^{4} \mathrm{~F}$ \\
\hline \multicolumn{11}{|l|}{$J=9 / 2$} \\
\hline 340251.58 & 340286.48 & -34.90 & 7 & 5 & 0.30 & 0.35 & 0.50 & $36 \%\left({ }^{1} \mathrm{G}\right)^{2} \mathrm{G}$ & $+26 \%\left({ }^{1} \mathrm{G}\right)^{2} \mathrm{H}$ & $+23 \%\left({ }^{3} \mathrm{~F}\right)^{4} \mathrm{~F}$ \\
\hline 330389.85 & 330344.67 & 45.18 & 8 & 8 & 0.32 & 0.33 & 0.40 & $43 \% \quad\left({ }^{3} F\right)^{2} G$ & $+27 \%\left({ }^{1} \mathrm{G}\right)^{2} \mathrm{G}$ & $+20 \%\left({ }^{3} \mathrm{~F}\right){ }^{4} \mathrm{~F}$ \\
\hline 318483.31 & 318446.42 & 36.89 & 7 & 6 & 0.24 & 0.20 & 0.30 & $35 \% \quad\left({ }^{3} \mathrm{~F}\right)^{4} \mathrm{~F}$ & $+31 \%\left({ }^{3} \mathrm{~F}\right)^{4} \mathrm{G}$ & $+21 \%\left({ }^{3} \mathrm{~F}\right)^{2} \mathrm{G}$ \\
\hline
\end{tabular}




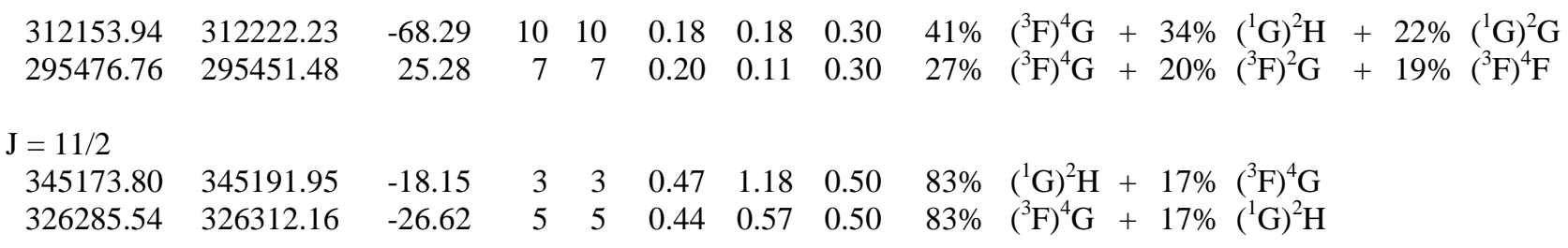


Table 4

Fitted and calculated parameter values $\left(\mathrm{cm}^{-1}\right)$ in the $5 \mathrm{~d}^{3}, 5 \mathrm{~d}^{2} 6 \mathrm{~s}$ and $5 \mathrm{~d} 6 \mathrm{~s}^{2}$ configurations of Pt VIII.

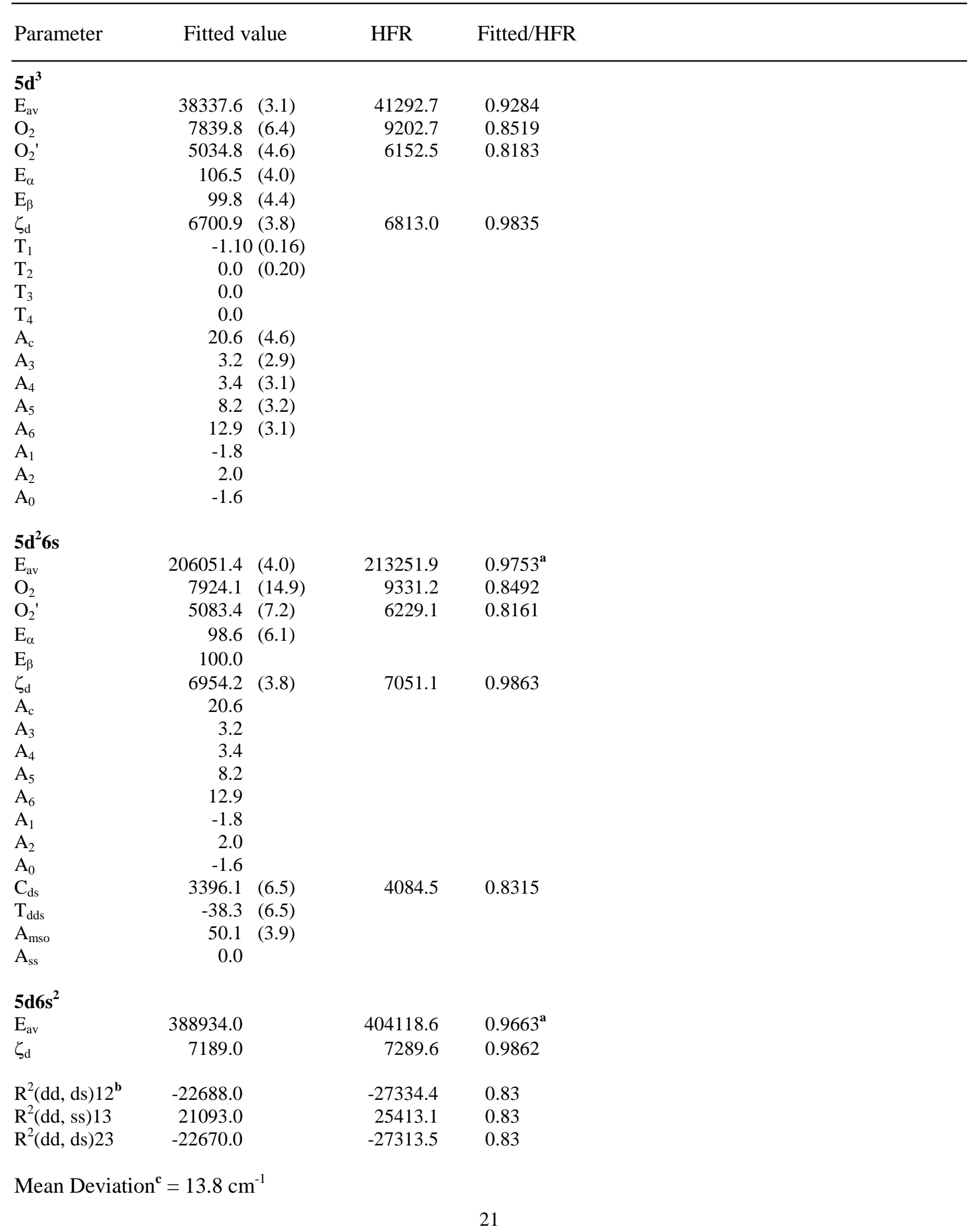


a The energy differences with respect to the ground configuration were used: $\left(\mathrm{E}_{\mathrm{av}}(\mathrm{conf})-\mathrm{E}_{\mathrm{av}}\left(5 \mathrm{~d}^{3}\right)\right)_{\mathrm{LSF}} /\left(\mathrm{E}_{\mathrm{av}}(\mathrm{conf})-\mathrm{E}_{\mathrm{av}}\left(5 \mathrm{~d}^{3}\right)\right)_{\mathrm{HFR}}$, where "conf" is either $5 \mathrm{~d}^{2} 6 \mathrm{~s}$ or $5 \mathrm{~d} 6 \mathrm{~s}^{2}$.

b Trailing digits xy in the $R$-integral labels denote interaction between configurations x and y, where 1 stands for $5 \mathrm{~d}^{3}, 2$ stands for $5 \mathrm{~d}^{2} 6 \mathrm{~s}$ and 3 stands for $5 \mathrm{~d} 6 \mathrm{~s}^{2}$.

c Mean Deviation $=\left[\left(\Sigma\left(\mathrm{E}_{\mathrm{obs}}-\mathrm{E}_{\text {calc }}\right)^{2}\right) /(\mathrm{n}-\mathrm{m})\right]^{1 / 2}$, where $\mathrm{n}$ is the number of known levels, $\mathrm{m}$ is the number of free parameters. 
Table 5

Fitted and calculated parameter values $\left(\mathrm{cm}^{-1}\right)$ in the $5 \mathrm{~d}^{2} 6 \mathrm{p}, 5 \mathrm{~d} 6 \mathrm{~s} 6 \mathrm{p}$ and $6 \mathrm{~s}^{2} 6 \mathrm{p}$ configurations of Pt VIII.

\begin{tabular}{|c|c|c|c|}
\hline Parameter & Fitted value & HFR & Fitted/HFR \\
\hline \multicolumn{4}{|l|}{$5 d^{2} 6 p$} \\
\hline $\mathrm{E}_{\mathrm{av}}$ & $320558.7 \quad(17.3)$ & 326520.3 & $0.9895^{\mathrm{a}}$ \\
\hline $\mathrm{O}_{2}$ & $7962.2 \quad(45.6)$ & 9362.5 & 0.8504 \\
\hline $\mathrm{O}_{2}^{\prime}$ & $5113.3(43.0)$ & 6247.5 & 0.8185 \\
\hline $\mathrm{E}_{\alpha}$ & 150.4 & & \\
\hline $\mathrm{E}_{\beta}$ & $87.4 \quad(40.1)$ & & \\
\hline$\zeta_{\mathrm{d}}$ & $7031.8 \quad(19.6)$ & 7104.7 & 0.9897 \\
\hline $\mathrm{A}_{\mathrm{c}}$ & 20.6 & & \\
\hline $\mathrm{A}_{3}$ & 3.2 & & \\
\hline $\mathrm{A}_{4}$ & 3.4 & & \\
\hline $\mathrm{A}_{5}$ & 8.2 & & \\
\hline $\mathrm{A}_{6}$ & 12.9 & & \\
\hline $\mathrm{A}_{1}$ & -1.8 & & \\
\hline $\mathrm{A}_{2}$ & 2.0 & & \\
\hline $\mathrm{A}_{0}$ & -1.6 & & \\
\hline $\mathrm{C}_{1}(\mathrm{dp})$ & $3900.8 \quad(29.6)$ & 4557.9 & 0.8558 \\
\hline $\mathrm{C}_{2}(\mathrm{dp})$ & $2787.4 \quad(32.6)$ & 3575.0 & 0.7797 \\
\hline $\mathrm{C}_{3}(\mathrm{dp})$ & $1429.4 \quad(34.9)$ & 1726.9 & 0.8277 \\
\hline $\mathrm{S}_{1}(\mathrm{dp})$ & $166.0 \quad(26.8)$ & & \\
\hline $\mathrm{S}_{2}(\mathrm{dp})$ & $-80.5 \quad(43.0)$ & & \\
\hline $\mathrm{T}_{16}$ & -25.0 & & \\
\hline $\mathrm{T}_{25}$ & 5.0 & & \\
\hline $\mathrm{T}_{26}$ & -35.0 & & \\
\hline $\mathrm{T}_{27}$ & 5.0 & & \\
\hline $\mathrm{T}_{28}$ & 55.0 & & \\
\hline $\mathrm{T}_{29}$ & -35.0 & & \\
\hline $\mathrm{T}_{30}$ & 10.0 & & \\
\hline$\zeta_{p}$ & 21133.9 & 19603.7 & 1.0781 \\
\hline $\mathrm{SS}_{02}$ & 0.0 & & \\
\hline $\mathrm{SS}_{20}$ & 0.0 & & \\
\hline $\mathrm{S}_{\mathrm{d}} \cdot \mathrm{L}_{\mathrm{p}}$ & -120.0 & & \\
\hline$S_{p} \cdot L_{d}$ & -17.0 & & \\
\hline $\mathrm{Z}_{\mathrm{pp}}^{2}$ & -50.0 & & \\
\hline $\mathrm{Z}_{\mathrm{dd}}^{2 \mathrm{P}}$ & 60.0 & & \\
\hline $\mathrm{Z}_{\mathrm{pp} \mathrm{p}^{\prime}}^{1}$ & 155.0 & & \\
\hline $\mathrm{Z}_{\mathrm{dd}^{1}}^{\mathrm{p}^{\mathrm{p}}}$ & 0.0 & & \\
\hline $\mathrm{Z}_{\mathrm{pp}}^{3}$ & 80.0 & & \\
\hline $\mathrm{Z}_{\mathrm{dd}}^{3}$ & -19.0 & & \\
\hline \multicolumn{4}{|l|}{$5 d 6 s 6 p$} \\
\hline $\mathrm{E}_{\mathrm{av}}$ & 499601.0 & 509589.5 & $0.9850^{\mathrm{a}}$ \\
\hline$\zeta_{\mathrm{d}}$ & 7282.0 & 7341.1 & 0.9919 \\
\hline $\mathrm{C}_{\mathrm{ds}}$ & 3382.0 & 4074.6 & 0.8300 \\
\hline $\mathrm{A}_{\mathrm{mso}}$ & 43.0 & & \\
\hline $\mathrm{A}_{\mathrm{ss}}$ & 0.0 & & \\
\hline $\mathrm{C}_{1}(\mathrm{dp})$ & 3991.0 & 4688.4 & 0.8512 \\
\hline $\mathrm{C}_{2}(\mathrm{dp})$ & 2835.0 & 3629.6 & 0.7811 \\
\hline $\mathrm{C}_{3}(\mathrm{dp})$ & 1443.0 & 1741.3 & 0.8287 \\
\hline $\mathrm{S}_{1}(\mathrm{dp})$ & 151.0 & & \\
\hline $\mathrm{S}_{2}(\mathrm{dp})$ & -65.0 & & \\
\hline
\end{tabular}




\begin{tabular}{|c|c|c|c|}
\hline$\zeta_{\mathrm{p}}$ & 22461.0 & 20894.4 & 1.0750 \\
\hline $\mathrm{S}_{\mathrm{d}} \cdot \mathrm{L}_{\mathrm{p}}$ & -120.0 & & \\
\hline $\mathrm{S}_{\mathrm{p}} \cdot \mathrm{L}_{\mathrm{d}}$ & -17.0 & & \\
\hline $\mathrm{Z}_{2 \mathrm{pp}^{\prime}}^{5}$ & -50.0 & & \\
\hline $\mathrm{Z}_{\mathrm{dd}^{\prime}}^{\mathrm{PP}}$ & 60.0 & & \\
\hline $\mathrm{Z}_{\mathrm{pp} p^{\prime}}^{1}$ & 155.0 & & \\
\hline $\mathrm{Z}_{\mathrm{dd}^{\prime}}^{1^{1}}$ & 0.0 & & \\
\hline $\mathrm{Z}_{\mathrm{pp} p^{\prime}}^{3}$ & 80.0 & & \\
\hline $\mathrm{Z}_{\mathrm{dd} \mathrm{d}^{\prime}}$ & -19.0 & & \\
\hline $\mathrm{C}_{\mathrm{sp}}$ & 14982.0 & 18050.2 & 0.83 \\
\hline $\mathrm{A}_{\mathrm{mso}}(\mathrm{sp})$ & -783.0 & & \\
\hline \multicolumn{4}{|l|}{$6 s^{2} 6 p$} \\
\hline $\mathrm{E}_{\mathrm{av}}$ & 693966.0 & 711872.0 & $0.9777^{\mathrm{a}}$ \\
\hline$\zeta_{\mathrm{p}}$ & 23945.0 & 22274.2 & 1.0750 \\
\hline $\mathrm{R}^{2}(\mathrm{dd}, \mathrm{ds}) 12^{\mathrm{b}}$ & -22561.3 & -27182.3 & 0.83 \\
\hline $\mathrm{R}^{2}(\mathrm{dp}, \mathrm{sp}) 12$ & -23153.4 & -27895.7 & 0.83 \\
\hline $\mathrm{R}^{1}(\mathrm{dp}, \mathrm{ps}) 12$ & -19517.0 & -23514.4 & 0.83 \\
\hline $\mathrm{R}^{2}(\mathrm{dd}, \mathrm{ss}) 13$ & 21040.0 & 25349.4 & 0.83 \\
\hline $\mathrm{R}^{2}(\mathrm{dp}, \mathrm{sp}) 23$ & -23356.8 & -28140.7 & 0.83 \\
\hline $\mathrm{R}^{1}(\mathrm{dp}, \mathrm{ps}) 23$ & -19594.2 & -23607.5 & 0.83 \\
\hline
\end{tabular}

Mean Deviation $^{\mathrm{c}}=116 \mathrm{~cm}^{-1}$

a The energy differences with respect to the ground configuration were used: $\left(\mathrm{E}_{\mathrm{av}}(\mathrm{conf})-\mathrm{E}_{\mathrm{av}}\left(5 \mathrm{~d}^{3}\right)\right)_{\mathrm{LSF}} /\left(\mathrm{E}_{\mathrm{av}}(\mathrm{conf})-\mathrm{E}_{\mathrm{av}}\left(5 \mathrm{~d}^{3}\right)\right)_{\mathrm{HFR}}$, where "conf" is either $5 \mathrm{~d}^{2} 6 \mathrm{p}, 5 \mathrm{~d} 6 \mathrm{~s} 6 \mathrm{p}$ or $6 \mathrm{~s}^{2} 6 \mathrm{p}$.

b Trailing digits xy in the $R$-integral labels denote interaction between configurations x and $\mathrm{y}$, where 1 stands for $5 d^{2} 6 p, 2$ stands for $5 d 6 s 6 p$ and 3 stands for $6 s^{2} 6 p$.

c Mean Deviation $=\left[\left(\Sigma\left(\mathrm{E}_{\mathrm{obs}}-\mathrm{E}_{\mathrm{calc}}\right)^{2}\right) /(\mathrm{n}-\mathrm{m})\right]^{1 / 2}$, where $\mathrm{n}$ is the number of known levels, $\mathrm{m}$ is the number of free parameters. 